\title{
THE LIMITING OF MAGNETOSPHERIC CONVECTION BY \\ DISSIPATION IN THE IONOSPHERE*
}

\author{
Edward Walbridge ${ }^{\neq}$ \\ Enrico Fermi Institute for Nuclear Studies \\ and Department of Physics \\ University of Chicago \\ Chicago, Illinois 60637
}

Laboratory for Astrophysics and Space Research

EFINS Preprint No. 67-29

April 1967

*This research was supported in part by the National Aeronautics and Space Administration under Grant No. NASA-NsG 96-60.

${ }^{*} A$ thesis submitted to the Department of Physics, the University of Chicago, Chicago, Illinois, in partial fulfillment of the requirements for the Ph.D. degree. 


\title{
THE LIMITING OF MAGNETOSPHERIC CONVECTION BY
}

\section{DISSIPATION IN THE IONOSPHERE*}

\author{
Edward Walbridge ${ }^{*}$ \\ Enrico Fermi Institute for Nuclear Studies \\ and Department of Physics \\ University of Chicago \\ Chicago, Illinois 60637
}

\begin{abstract}
The constant of proportionality between the magnetospheric convection
\end{abstract} velocity and the magnetic stressing required to produce that velocity is obtained. This constant, the drag coefficient, is independent of the mechanism which drives the convection. From the drag coefficient the rate of dissipation due to joule and viscous heating is obtained as a function of the convection velocity. The driving mechanism must supply at least as much energy as is dissipated. It is found that the maximum rate at which the solar wind can do work on the magnetospheric plasma is much greater than is required for supplying the energy dissipated, even with convection velocities up to about $1 \mathrm{~km} / \mathrm{sec}$ (in the ionosphere). Given velocities as high as $1 \mathrm{~km} / \mathrm{sec}$, a number of interesting consequences follow. The role of viscosity in the dynamics of the ionosphere is assessed. It is found that viscous dissipation is usually not significant. The effect of neutral gas motions on the joule dissipation is considered.

*This research was supported in part by the National Aeronautics and Space Administration under Grant No. NASA-Ns G 96-60.

${ }^{\ddagger}$ A thesis submitted to the Department of Physics, the University of Chicago, Chicago Illinois, in partial fulfillment of the requirements for the Ph.D. degree. 


\section{i. Introduction}

The earth's magnetosphere is a region of high electrical conductivity; thus geomagnetic field lines are 'frozen' into its ambient plasma. Similarly, geomagnetic field lines are frozen into the conducting earth. Between the conducting earth and the conducting magnetosphere lies a layer of non-conducting neutral atmosphere. Within this insulating layer the field lines are not frozon in so that, as pointed out by Gold (1959), the magnetosphere may move relative to the earth.

It was pointed out by Axford and Hines (1961) that: if the polar DS current system is driven from above there must be large scale convection. It is easy to see why the DS system indicates magnetospheric convection. There must be an electric field $\vec{E}$ to drive the currents through the ionosphere. The electrical conductivity along the magnetic field is so great that the magnetic field lines are essentially equipotential lines. Thus out in the magnetosphere the same potential differences must exist between the lines of force as are inferred from the currents in the ionosphere. In the magnetosphere an electric field implies a velocity

$$
\overrightarrow{V_{D}}=c \frac{\vec{E} \times \vec{B}}{B^{2}}
$$

Here $\quad \vec{V}$ is the drift velocity, $\vec{B}$ the geomagnetic field, and $C$ the velocity of light. The pattern of such convective flow can be found from the DS current pattern by upward mapping of the electric field.

This pattern of convection and its consequences were examined by Axford and Hines (1961). They estimate that the convective velocity at ionospheric heights

1 We note that dynamo mechansims, which generate the DS system by means of ionospheric winds, also lead to convection (Hines, 1964). 
may be as high as $1 \mathrm{~km} / \mathrm{sec}$ and that the convection may be responsible for much of the aurora, the trapped radiation, and the growth and decay of the main phase of magnetic storms. Hence it is important to understand the physics of convection. The Axford and Hines convection model is indicated in Fig. 1. Each tube of flux above the nonconducting atmosphere moves as a unit because it is embedded in a conducting medium. In their model the plasma in the outer magnetosphere convects away from the sun, returning through the interior. In this convective process all the flux tubes above a certain latitude take part in the motion. The path followed by the feet of the flux tubes in the ionosphere is shown in Fig. 2. The feet of the flux tubes drag the viscous, dissipative ionosphere around with them, so that a driving force is needed to maintain the convection. While the existence of some driving mechanism is required, no particular mechanism is crucial. The convection suggested by Axford and $H$ ines can be considered quite independently of any mechanism which drives it.

The solar wind may be the ultimate source of driving energy for the convection, with energy being transferred into the magnetosphere by any of various mechanisms. For instance, Axford and Hines (1961) suggested a "viscous-like" interaction between the solar wind and the magnetospheric material. An alternative process involves the reconnection of field lines and has been examined by Levy, Petschek, and Siscoe (1963).

On the other hand, the energy required to drive the convection could come from a source distinct from the solar wind. For example, Fejer (1964) has found that the interaction of magnetospheric rotation with a belt of energetic protons can lead to a DS-like current system. Associated with the $\vec{E}$ fields of this system there must be an Axford and Hines type convection pattern. 
But no matter what the driving mechanism, a driving force is required in order to maintain convection against the friction of the dissipation region in the ionosphere. Without worrying about the driving mechanism we will investigate the physics of convection in the dissipation region, with a view to finding the constant of proportionality between the driving force and the convective velocity. From this proportionality constant we will obtain, as a function of the convection velocity, the ionospheric dissipation rate due to joule and viscous heating. Some driving mechanism must supply the energy at this rate in order to maintain the convection. Once we have thus found the energy input required to maintain a certain convection rate, e.g. $1 \mathrm{~km} / \mathrm{sec}$ at ionospheric levels, we will be able to ask if a given driving mechanism can provide that input, thereby producing the convection and hence the aurora, trapped radiation, etc. In particular, we can ask if the solar wind can supply energy at the required rate (no matter how that energy is transferred into the magnetosphere).

\section{General Considerations}

In order to study the convective process at ionospheric levels, we adopt a model. But before describing and disucssing this model, we can discuss the real-life process from which the model is extracted. The convecting ionosphere is a complex system; seen by an observer on the earth, the pattern of convection varies in time, the ions, electrons and neutrals which compose the ionosphere can all drift at different velocities, and the conductivity is a tensor instead of a scalar. We wish to distill from this complex situation the essential physics of convection at ionospheric levels.

Now convection is limited by joule and viscous dissipation occurring in the lower ionosphere. We want to find out within what height range $z_{1} \leq z \leq z_{2}$ the joule and viscous dissipation takes place. (Throughout this paper $z$ refers to height 
above the ground.) With the dissipation region boundaries $z_{1}$ and $z_{2}$ established, the boundary conditions which are obtained there can be specified.

The Convective Process

Associated with the convective velocity $\overrightarrow{V_{P}}$ of the magnetospheric plasma there is an electric field such that

$$
\vec{E}+\overrightarrow{V_{p}} \times \vec{B} / C=0
$$

to good approximation. In that region of the magnetosphere where the driving mechanism is operative a driving force pushes (positive) charges in the direction of $-\vec{E}=$ $\vec{V} \times \vec{B} / C \quad$, that is, from lower to higher potential. For example, suppose that the driving mechanism operates in the outermost region of the magnetosphere; positive charges will be driven up the potential gradient from the point $Q$ toward the point $P$ in Fig. 1. From the neighborhood of $P$ charges will flow along $\vec{B}$ down to the northern and southern polar ionospheres, where there are frequent charged particleneutral collisions. In each such collision the guiding center is shifted from a higher potential field line to a lower potential line. The charges which traveled down to the ionosphere along the field line $P$ (i.e., the field line passing through point $P$ ) will move through the ionosphere in the direction of $\vec{E}$ toward the field line $Q$ and then back up to the region of the driving mechanism, thus completing the circuit. This latter region is thus a generator or source of E.M.F. The ionosphere acts as an electric motor because there currents set the neutral gas in motion.

These remarks indicate the essential physics of the convection process by 
means of a somewhat simplified picture. In full detail the situation is more complicated. In particular the current flow along $\vec{B}$ consists mainly of moving electrons, while in the ionosphere the currents (parallel to $\vec{E}$ ) result primarily from moving ions. In addition to the flow of charge in the ionosphere along $\vec{E}$, there will be a significant flow perpendicular to $\vec{E}$ (and $\vec{B}$ ), viz., the Hall current. This means that, if we think of the currents as consisting of moving positive charges, these charges will flow through the ionosphere, not just in the direction from the field line $P$ to the field line $Q$, but also perpendicular to that direction. Thus a charge which comes down along the field line $P$ will not travel back up to the region of the driving mechanism along the same field line as it would have without the Hall current. It will however return to the region of the driving mechanism, where driving forces are exerted to raise it again to a higher potential field line. Hence the essential physics of this process has been captured in the simple discussion above, where Hall currents are ignored. The Convection Pattern at lonospheric Levels

Figure 2 shows the plasma flow pattern as viewed in a frame which does not rotate with the earth. The pattern of Fig. 2 represents the flow of the ion-electron plasma, not the full plasma including neutrals. At the heights for which Fig. 2 is drawn, the neutral gas does not in general move with the ion-electron gas. Henceforth when reference is made to the plasma, we mean the ion-electron plasma. We want to study convection from the standpoint of an observer rotating with the earth. Fig. 3 is obtained from Fig. 2 by subtracting out the earth's rotational velocity. At each point of Fig. 3 the velocity vector represents the velocity of convecting plasma as seen by an observer directly under that point.

An observer on the Earth will rotate under Figs. 2, 3. In Fig. 2 a blob of 
plasma convects around in one of the closed loops (provided a steady state exists). As viewed from an earthbound frame, that is, a frame of reference rotating with the Earth, the path of this convecting blob will be in general a spiral which does not retrace itself. It will not in general be a closed loop.

The plasma convection velocity and the associated electric field observed directly above an observer rotating with the Earth will vary in time as that observer rotates under the fixed pattern of Figs. 2, 3. Thus for an earthbound frame the plasma velocity and the electric field are functions of time as well as position.

An observer on the Earth at the latitude of $A$ or $D$ in Fig. 2 will observe the plasma velocity above to vary with a period of 24 hours, that is with a time scale $t_{s}=24 \mathrm{hrs} .=0.864 \times 10^{5} \mathrm{sec}$. This is the longest time scale that can be associated with the plasma motions of Figs. 2,3. An observer on the Earth rotating under B in Fig. 2 will see the plasma which moves from $C$ to $B$ changing velocity with a time scale $t_{\mu}=1 \times 10^{4} \mathrm{sec}$. This is the shortest significant time scale that can be associated with the plasma motions. The time $t_{s}=0.864 \times 10^{5} \mathrm{sec}$ corresponds to a minimum angular frequency $\quad w=0.7 \times 10^{-4} \mathrm{sec}^{-1}$ and $t_{\Lambda}=10^{4} \mathrm{sec}$ corresponds to a maximum frequency $w=6 \times 10^{-4} \mathrm{sec}^{-1}$. These two $w$ values define a range of frequencies $0.7 \times 10^{-4} \sec ^{-1} \leq \omega \leq 6 \times 10^{-4} \mathrm{sec}^{-1}$. In the model the plasma velocity will be taken to vary as sin $\omega t$ with $\omega$ taken from this range. Ion, Electron, and Neutral Gas Motions

Above $z=300 \mathrm{~km}$, the ion gas velocity $\overrightarrow{V_{i}}$ satisfies the equation:

$$
\vec{E}+\overrightarrow{V_{i}} \times \vec{B} / c=0
$$


to 1 percent or better. For $z \leq 300 \mathrm{~km}$, ion-neutral collisions prevent (2) from being well satisfied. But the equation

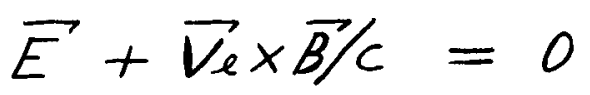

for the electron gas velocity $\overrightarrow{V_{e}}$ is satisfied to good approximation down to $z=100 \mathrm{~km}$.

From (3) it follows that $\vec{E}$ is perpendicular to $\vec{B}$ down to $z=100 \mathrm{~km}$. For $z \geq 300 \mathrm{~km}$, both (2) and (3) are satisfied so that ions and electrons will move at essentially the same velocity, the plasma velocity $\overrightarrow{V_{p}}$. (We do not define $\quad \overrightarrow{V_{p}}$ for the range $z<300 \mathrm{~km}$ because the ions and electrons do not move together in that range. Also it is clear from (2) and (3) that $\overrightarrow{V_{i}}$ and $\overrightarrow{V_{e}}$ can differ by a component along $\vec{B}$, but this will be small compared to $\left|\overrightarrow{V_{i}}\right|$ or $\left|\overrightarrow{V_{e}}\right|$.) Then $\overrightarrow{V_{p}}$ will satisfy Eqn. (1) for $z \geq 300 \mathrm{~km}$. The magnetic field lines will move with the plasma for $z \geq 300 \mathrm{~km}$ and with the electrons for $z \geq 100$ km. Figs. 2,3 show the velocity $\overrightarrow{V_{p}}$ for $z \geq 300 \mathrm{~km}$ in the ionosphere (or $\overrightarrow{V_{e}}$ for $z \geq 100 \mathrm{~km})$

The ionosphere consists primarily of neutral gas. Due to collisions this gas tends to convect $\left(\overrightarrow{V_{N}}\right)$ with the ions, especially at higher levels where the neutral density is small. The neutrals convect closely with the ions above $250 \mathrm{~km}$ for motions with characteristic frequency $\quad W=0.7 \times 10^{-4} \mathrm{sec}^{-1}$. But they do not move with the ions at any level for $\omega=0.6 \times 10^{-3} \mathrm{sec}^{-1}$. The Current Density and Dissipation

The current density $\vec{y}$ in the ionosphere is given by 


$$
\vec{\gamma}=\sigma_{p}\left(\overrightarrow{E_{\perp}}+\vec{V}_{N} \times \vec{B} / c\right)+\sigma_{H}\left(\overrightarrow{E_{\perp}}+\overrightarrow{V_{N}} \times \vec{B} / c\right) \times \vec{B} /|\vec{B}|+\sigma_{11} \overrightarrow{E_{11}}
$$

The symbols $\perp$ and $\|$ denote respectively components perpendicular and parallel to $\vec{B}$. $\sigma_{P}$ is the Pederson conductivity, $\sigma_{H}$ the Hall conductivity, and $\sigma_{11}$ the conductivity parallel to $\vec{B} \quad 2$

The joule dissipation rate per unit volume is given by the scalar product of $\vec{\gamma}$ with the electric field $\vec{E}^{\prime}=\vec{E}+\vec{V}_{N} \times \vec{B} / C \quad$ seen in a frame of reference moving with the velocity $\vec{V}_{N}$. Thus the joule dissipation is

$$
\vec{\gamma} \cdot \vec{E}^{\prime}=\sigma_{p}\left(\overrightarrow{E_{\perp}}+\vec{V}_{N} \times \vec{B} / c\right)^{2}+\sigma_{11} \overrightarrow{E_{11}^{2}}=\gamma_{p}^{2} / \sigma_{p}+\gamma_{11}^{2} / \sigma_{11}
$$

2

For the most part electrostatic units are used in this paper, but it is often convenient to give conductivities in EMU. When EMU are used, it will be explicitly stated; otherwise ESU are to be understood. We note that $\sigma(E S U)=C^{2} \sigma(E M U)$ We have defined $\quad \sigma_{H}$ so that the Hall current is $\vec{y}_{H}=$

$$
\sigma_{H}\left(\overrightarrow{E_{\perp}}+\overrightarrow{V_{N}} \times \vec{B} / C\right) \times \vec{B} /|\vec{B}|
$$

is opposite in sign (being negative) to the

$$
\sigma_{H} \vec{B} /|\vec{B}| \times\left(\overrightarrow{E_{\perp}}+\overrightarrow{V_{N}} \times \vec{B} / C\right)
$$

- Thus the $\sigma_{A}$ used here $\sigma_{H}$ used when $\quad \vec{J}_{H}=$

- The conductivities we use are taken primarily from Johnson (1961) as are also mass densities and other data. Those conductivities are for mid-latitudes. It should be noted that Chapman (1956) suggests lower conductivities in the polar ionosphere. Our final conclusions are not altered if lower conductivities are appropriate. So we shall use mid-latitude conductivities. 
where $\quad \vec{f}_{p}=\sigma_{p}\left(\vec{E}_{\perp}+\vec{V}_{N} \times \vec{B} / C\right)$

is the Pederson current

and

$$
\vec{j}_{11}=\sigma_{11} \overrightarrow{E_{11}}
$$

is the current parallel to

$\vec{\beta}$

- It should

be noted that (5) is independent of

We are interested in finding the upper boundary $\left(z=z_{2}\right)$ and the lower boundary $\left(z=z_{1}\right)$ of the region (the dissipation region) with in which essentially all the joule and viscous dissipation occurs. We have estimated $\vec{y} \cdot \vec{E}^{\prime}$ at various levels and find that to fairly good approximation (within 5 percent) all the joule dissipation occurs in the range $100 \mathrm{~km} \leq z \leq 300 \mathrm{~km}^{3}$ (essentially because $\sigma p$ is small outside this range).

So we need consider $\quad \vec{y} \cdot \vec{E}^{\prime}$

$300 \mathrm{~km}$. In this range the condition

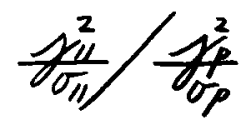
only in the range $100 \mathrm{~km} \leq z \leq$ so that the joule dissipation depends primarily on $\sigma_{p}$. It is not hard to demonstrate this. Charge will flow down into the lower ionosphere as $\vec{y}_{\prime \prime} \quad$ through a horizontal area $A_{\|}$with unit normal parallel to $\vec{B}$ and then will flow perpendicularly to $\vec{B}$ in the lower ionosphere as $\overrightarrow{\gamma_{P}}$ through an area $A_{p}$ with unit normal in the direction of $\overrightarrow{F_{p}}$. Conservation of current requires that

$$
\left|F_{11}\right| A_{\|}=\left|F_{p}\right| A_{p} .
$$

3

This range is extended upwards perhaps another $200 \mathrm{~km}$ for conditions of night-time, sunspot maximum. When we refer herein to daytime or night-time, sunspot maximum or minimum conditions, we mean the conditions of conductivity $\sigma_{p}(z), \sigma_{\mu}(z)$ and $V_{11}(z)$, electron density, etc., as defined for these times in Hanson (1961). 
The thinness of the region where there is significant $(200 \mathrm{~km})$ means that

$$
A_{\|} \geq A_{p} \quad \text { so that }\left|\vec{y}_{11}\right| /\left|\overrightarrow{j_{p}}\right|=A_{P} / A_{\|} \leq 1 \text {. }
$$

Further $\sigma_{p} / \sigma_{\|}<<1$ is satisfied for $z \geq 100 \mathrm{~km}$. Hence 4

$\frac{y_{111}^{2}}{\sigma_{11}} / \frac{y_{p}^{2}}{\sigma_{p}^{2}}=\left(A_{p} / A_{11}\right)^{2}\left(v_{p} / v_{11}\right)<<1$

for $100 \mathrm{~km} \leq z \leq 300 \mathrm{~km}$.

In general, (6) is satisfied in the region where $\vec{y} \cdot \vec{E} \cdot \quad$ is significant, so we may write for that region

$$
\vec{y} \cdot \overrightarrow{E^{\prime}}=y_{p}^{2} / \sigma_{p}=\sigma_{p}\left(\overrightarrow{E_{\perp}}\right)^{2}=\sigma_{p}\left(\overrightarrow{E^{\prime}}\right)^{2}
$$

By substituting $\quad \vec{y}_{11}=\sigma_{11} \vec{E}_{\|} \quad$ and $\quad \overrightarrow{y p}_{p}=\bar{E}_{\perp}$

into (6), it is seen that $|\vec{E} \| / /| E_{\perp} \mid<<1$ down to at least $z=100 \mathrm{~km}$. This conclusion was earlier drawn on the basis of (3).

In this section we have specified the height range within which essentially all the joule dissipation occurs. In the next section we determine the height range within which the viscous dissipation takes place. Then it will be possible to define the boundaries $z_{1}$ and $z_{2}$ of the dissipation region.

Viscous Dissipation

In order to discuss viscous dissipation it is necessary to know something about

${ }^{4}$ This discussion tacitly assumes that $\nabla \cdot \overrightarrow{Y_{H}}=0$, but if this is not the case and much of the charge which flows in as $/ \vec{y}_{11} / A_{\|} \quad$ leaves as Hall current, then similar arguments again yield (6). 
$\overrightarrow{V_{N}}$ as a function of $z$. (Henceforth the subscript $N$ is dropped so that $\overrightarrow{V_{N}} \rightarrow \vec{V} \quad$.) We have investigated the neutral gas motions by considering $\vec{V}$ to be driven solely by $\vec{y} \times \vec{B} / C \quad$ forces. It is thus found that $\quad|\vec{V}(z \leq 100 \mathrm{~km}) / /| \overrightarrow{V_{D}} \mid=0$ to fairly good approximation (less than 6 percent), so that below $100 \mathrm{~km}$ the neutrals are immobile with respect to the earth. This is because, for $z \leq 100 \mathrm{~km}$, the ionosphere is too massive and the sinusoidally varying force $\quad \vec{y} \times B / C \quad$ too weak, to produce a significant $\vec{V}$. Since $\quad \vec{V} \approx 0$ below $100 \mathrm{~km}$, there is negligible viscous dissipation $\mu(\partial \vec{V} / \partial z)^{2} \quad$ below that level. ${ }^{5}(\mu$ denotes the coefficient of viscosity.)

Thus $z=100 \mathrm{~km}$ can be taken as the base $z_{1}$ of the dissipation region above which essentially all the joule and viscous dissipation occurs. The boundary condition at $100 \mathrm{~km}$ is $\quad \vec{V}\left(z_{1}\right)=0$.

The above conclusions were found by neglecting viscous forces $(\mu=0)$. We have estimated these forces and found that they have little effect in the vicinity of $100 \mathrm{~km}$ and below. Thus viscous forces do not alter the above conclusions.

Again by taking $\quad \vec{V}$ to be determined solely by the force $\quad \vec{y} \times \vec{B} / C$ and estimating the additional effect of viscosity it is found that viscous dissipation gen-

${ }^{5}$ Further, $\quad \vec{V}$ varies slowly with $z$ below $100 \mathrm{~km}$. The general viscous stress tensor for an ion-neutral gas in the presence of a magnetic field is discussed in Appendix 1. The behavior of $\mu$ with $z$ is also given. 
erally occurs below $300 \mathrm{~km}$. ${ }^{6}$ Thus the boundaries of the dissipation region are $z_{2}=300 \mathrm{~km}$ (occasionally higher) and $z_{1}=100 \mathrm{~km}$. Within this region the dissipation rate per unit volume is $\quad d \varepsilon / d t=\sigma_{p}\left(\overline{E^{\prime}}\right)^{2}+R_{i j}\left(\partial V_{i} / \partial x_{j}\right)$ where $R_{i j}$ is the viscous stress tensor (Appendix 1).

III. The Model

To consider the problem in its real-life detail would unnecessarily complicate it and render it intractable. Instead, we consider a simplified model to represent the convection phenomenon. This is shown in Figs. 4, 5. The model contains the essential features of the convective process.

The rationale for the model is the following. An observer on the rotating Earth will see plasma motions above, which vary in a periodic manner. Let the $z$ axis of a cartesian coordinate system lie along the observer's vertical. At time $t_{0}$ let the $y$ axis lie in the direction of the plasma velocity $\overrightarrow{V_{p}}$ seen directly above the observer. Then by eqation (1) the $x$ axis will be parallel to $\vec{E}$ (if $\vec{B}$ is vertical). $\quad \overrightarrow{V_{P}}$ and $\vec{E}$ will vary in time with respect to this local coordinate system defined at $t=t_{0}$. The model is an idealization of the convection as viewed in this local coordinate system.

${ }^{6}$ An exception to this occurs for $\quad \omega=0.6 \times 10^{-3} \mathrm{sec}^{-1}$ and daytime, sunspot maximum conditions (Figs. 8, 10) when there can be significant viscous dissipation up to $340 \mathrm{~km}$. As determined by the $\vec{\gamma} \times \vec{B} / C \quad$ force alone $\vec{V}$ is found to be almost independent of $z$ above a certain critical level $z_{c}$. With viscous forces included $\vec{V}$ is essentially independent of $z$ above $z_{c}+D_{v}\left(z_{c}\right)$ (where $D_{v}(z)=(\mu(z) / \rho(z) w)^{\frac{1}{2}}$ and $\rho$ is the neutral gas mass density)and thus there is negligible viscous dissipation above this level. 
In this model the earth is taken to be flat and there is translational symmetry along the $y$ axis.

The model is divided into five regions.

Region 5 represents the upper magnetosphere where some mechanism pushes charges from a low potential region (the right side of Fig. 4) to a high potential region (the left side of Fig. 4).

Region 4 represents the region of the magnetosphere through which momentum is transferred by magnetic stresses from Region 5 to Region 3. In this region the neutral gas convects rigidly and the field is frozen to the plasma.

Region 3 represents the dissipation region which lies in the range $z_{1}=100 \mathrm{~km}$ $\leq z \leq z_{2}$. Usually we have $z_{2}=300 \mathrm{~km}$.

Region 2 includes part of the ionosphere (from $100 \mathrm{~km}$ to $60 \mathrm{~km}$ ) and the entire non-conducting a tmosphere down to $z=0$.

Region 1 represents the earth.

The velocity $\overrightarrow{V_{p}}$ is taken to lie along the $y$ axis of the model, $\overrightarrow{V_{p}}=V_{p y}(z) \hat{y} \quad$. By collisions the plasma particles establish a neutral gas velocity parallel to $\overrightarrow{V_{p}}$ so that we take $\vec{V}=V_{y}(z) \hat{y}$ The unperturbed magnetic field is $\overrightarrow{B_{0}}=-B_{0} \hat{z}=$ constant and the electric field, above $100 \mathrm{~km}$, is $\vec{E}=E_{x_{0}} \hat{x}$

$$
E_{x 0} \text { is constant in space but varies in time as sinet. With }
$$
these specifications the current density (4) becomes for $z \geq 100 \mathrm{~km}$ :

$$
\vec{y}=\sigma_{p}\left(E_{x_{0}}-v_{y} B_{0} / c\right) \hat{x}+\sigma_{\mu}\left(E_{x_{0}}-v_{p} B_{0} / c\right) \hat{y}
$$


where the conductivities $\sigma_{p}$ and $\sigma_{H}$ are assumed to depend only on $z$. The current flow is indicated in Figs. 4, 5. Vertical currents flow along the right and left boundaries of the model (Fig. 4). The $x$ and $z$ direction currents (Fig. 4) constitute current loops. A positive charge will move around one of these loops, from the low to the high potential boundary in Region 5, down the high potential boundary to Region 3, then across to the low potential boundary again and back up. In reality, of course, the vertical currents are not restricted to two sheets. But we are not interested in the vertical current structure; rather we are interested in $\vec{y}_{p}(z)=y_{x}(z) \hat{x}$ which is well represented by the model.

Three matters relating to the model require discussion: 1) the electric field below $100 \mathrm{~km}$; 2) the perturbation magnetic field; and 3) the equations of motion. 1) The Electric Field Below $100 \mathrm{~km}$.

\section{The Condition}

\section{$\left|E_{11}\right| /\left|E_{+}\right| \quad<<1$ is well satisfied above $100 \mathrm{~km}$ but will not} hold at most levels below $100 \mathrm{~km}$. The character of the $\vec{E}$ field below $\mathrm{z}_{1}$ is indicated in Fig. 4. Since the earth is highly conducting, charges will move within it to cancel out any field $\vec{E}$ imposed from above and confine it to $z \geq 0$. Viewed from the earth the field $\vec{E}$ varies in time. But the conductivity of the solid earth is so high that $\vec{E}$ and any associated changes in $\vec{B}$ penetrate but little into the earth's interior.

Fig. 4 shows the $\vec{E}$ field configuration taking into account the fact that the earth's surface is an equipotential surface. The field for the region $0 \leq z \leq z_{1}$ was found by solving the equation $\nabla^{2} U=0$ (where $\vec{E}=-\nabla U$ ) subject to boundary conditions: 


$$
\begin{aligned}
& E_{x}\left(x, z=z_{1}\right)=E_{x 0}=\text { constant in space } \\
& E_{x}(x, z=0)=0 .
\end{aligned}
$$

The solution is

$$
u=-\left(E_{x} / z_{1}\right) \times z \text {. }
$$

From equation (1) we have that

$$
E_{x_{0}}=V_{p y}\left(z \geq z_{z}\right) B_{0} / c
$$

With, for example, $\quad V_{p y}\left(z \geq z_{2}\right)=1 \mathrm{~km} / \mathrm{sec}$ and $\quad L=400 \mathrm{~km}$ (corresponding to the high velocity region $E, F$ or $G, H$ in Fig. 3) and $B_{0}=.5$ gauss, the potential drop across the width $L$ of the model is $2 \times 10^{4}$ volts. We expect that Fig. 4 gives a good qualitative idea of real-life $\vec{E}$ field behavior at the lowest levels. In particular we expect that in the actual ionosphere-atmosphere system $\left|\overrightarrow{E_{\perp}}\right|$ will diminish and $|\overrightarrow{E \prime \prime}|$ increase with decreasing altitude. In the actual system however the $\vec{E}$ field will be roughly horizontal down to $80 \mathrm{~km}$, and there will not be a discontinuity at $z_{1}=100 \mathrm{~km}$.

2) The Perturbation Magnetic Field

The current $\vec{\gamma}$ produces a perturbation field which is small compared to $\overrightarrow{B_{0}}$ and which must be added to it to obtain the full geomagnetic field $\vec{B}$. It is convenient to divide the perturbation field into two components so that $\vec{B}=$ $-B_{0} \hat{z}+b_{y} \hat{y}+\overrightarrow{b_{N}}$. The sources of the perturbation component $b_{y} \hat{y}$ are the $x$ and $z$ direction currents. The $x, z$ current system of the model is just the current system of an infinitely long solenoid. The field $b_{y} \hat{y}$ does not extend to
the earth. In addition to $b_{y} \hat{y}$ there is a portion $b_{H}$ of the perturbation due to the Hall currents and a current system in the earth $\left(\nabla \times \vec{b}=(4 \pi / c) \overrightarrow{j H}^{H}\right.$ 
in the ionosphere). The earthbound current system is such that $\vec{b}_{A}$ is confined primarily to regions 2 and 3 . It is the perturbation field $\vec{b}$ which, observed on the ground, leads to the deduction of the DS current system.

We are interested in finding the magnetic stress $\quad S\left(z_{2}\right)$ required to produce a given convection rate. It is convenient to measure the convection rate by the plasma velocity at $z=z_{2}, i_{0} e_{0} \quad V_{p y}\left(z=z_{2}\right)$. The stress $S\left(z_{2}\right)$ applied against the friction of the charged particle-neutral collisions results in

$$
V_{p y}\left(z_{2}\right) \text {. }
$$

Since the velocities $\overrightarrow{V_{P}}$ and $\vec{V}$ are taken to lie in the $y$ direction, the relevant stress tensor component is that giving the ty momentum transferred in the -z direction (per second per $\mathrm{cm}^{2}$ ), that is, $B_{z} B_{y} / 4 \pi=-B_{0} b y / 4 \pi$ so that $S\left(z_{2}\right)=-B_{0} b_{y}\left(z=z_{2}\right) / 4 \pi \quad\left(\overrightarrow{b_{H}}\right.$ has components only in the $x$ and $z$ directions). $\quad S\left(z_{2}\right)$ is the momentum flux down the field lines from the region of the driving mechanism. $\quad S\left(z_{2}\right)$ and $V / p y\left(z_{2}\right)$ are related by a constant of proportionality $C \operatorname{ExP}(i \theta)$, where $C$ is the drag coefficient and $\theta$ the phase difference. That is, $S\left(z_{2}\right)=C X$ $\operatorname{EXP}(i \theta) V_{P y}\left(z_{2}\right)$. We want to obtain numbers for the drag coefficient. The Hall current does not produce any dissipation so we henceforth ignore $\overrightarrow{J H}_{H}$ and $\vec{b}_{H}$. The field $-B_{0} \hat{z}+b_{y} \hat{y} \quad$ is indicated in Fig. 7. $b_{y}$ is a negative quantity.

3) The Equations of Motion

We wish to find the equations, appropriate to the model, for determining the functions $V_{y}(z, t)$ and $b_{y}(z, t)$. The basic equations are Equation (4) for $\vec{y}$ in terms of $\overrightarrow{E^{\prime}}$, the force equation, and Maxwell's 
equations. For the geometry of the model, (4) becomes (7). Neglecting $\vec{J}_{H}$ and $\vec{b}_{\mu}$, the equation $\vec{j}=(c / 4 \pi) \nabla \times \vec{B} \quad$ and Equation (7) together yield

$$
y_{x}=\sigma_{p}\left(E_{x_{0}}-v_{y} B_{0} / c\right)=-(c / 4 \pi)\left(\partial b_{y} / \partial z\right)^{7}
$$

This is one of the two equations needed to solve for $V_{y}$ and $b_{y}$.

The other is the force equation. We neglect the Coriolis force as it does no work. ${ }^{8}$ The model is independent of $y$ so that $\alpha p / \partial y=0$ where $p$ is the pressure, and $\left(d V_{y} / d t\right) \hat{y}=\left(\alpha V_{y} / J t\right) \hat{y}$. The Lorentz force is $\left.\overrightarrow{y_{p}} \times \overrightarrow{B_{0}} / c=\left(y_{x} B_{0} / c\right) \hat{y}=-\left(B_{0} / 4 \pi\right)(\alpha \log / \partial z) \hat{y}\right)$ and the viscous force is $\partial / \partial z(\mu(\partial V y / \partial z)) \hat{y}$ (with

$$
\begin{aligned}
& \mu=\mu(z)) \\
& \text { The neutral gas force equation, appropriate to the model, is then } \\
& \begin{aligned}
\rho\left(\partial V_{y} / \partial t\right) & =-\left(B_{0} / 4 \pi\right)\left(\partial b_{y} / \partial z\right)+\partial / \partial z\left(\mu\left(\partial V_{y} / \partial z\right)\right) \\
& =\partial / \partial z\left(-B_{0} b_{y} / 4 \pi+\mu\left(\partial V_{y} / \partial z\right)\right)
\end{aligned}
\end{aligned}
$$

${ }^{7}$ We note that the total electric field is actually $E_{x}=-V_{\text {ey }} B_{0} / c=$ $E_{x 0}+\Delta E(x ; z)$ where $\Delta E$ is the induction component of $E_{x}$, Exo being produced by space charge. $\Delta E$ is small compared to $E_{x 0}$, and we neglect it. Furthermore, $\Delta E$ varies little over the vertical dimension $\left(z_{2}-z_{1}\right)$ of the dissipation region. This is because the Alfven wavelength is much greater than $\left(z_{2}-z_{1}\right)$ ${ }^{8}$ Although the Coriolis force does no work directly, it does alter $\vec{V}$ and thus affects somewhat the joule and viscous dissipations. Similarly, although $\overrightarrow{f^{A}}$ does not by 
itself result in dissipation, the force significant.

The quantity $\left(-B_{0} b y / 4 \pi+\mu(\alpha V / \partial z)\right)$ is the total stress, magnetic plus viscous, exerted in the $+y$ direction through a unit area with normal in the $-z$ direction.

We take $\quad V_{p y}\left(z \geq z_{2}\right)=V_{p o} \sin u t$ with $V_{p o} a$ constant and with $w$ in the range $0.7 \times 10^{-4} \mathrm{sec}^{-1} \leq w \leq 0.6 \times 10^{-3} \mathrm{sec}^{-1}$. Equations (8), (9) and (10) may then be combined to yield

$$
\begin{aligned}
\alpha / \partial z\left(\mu\left(\partial V_{y} / \partial z\right)\right) & -\left(B_{0}^{2} \sigma_{p} / c^{2}\right) V_{y}-\rho\left(\partial V_{y} / \partial t\right) \\
= & -\left(B_{0}^{2} \sigma_{p} / c^{2}\right) V_{p o} \text { sinut }
\end{aligned}
$$

The solution to this equation can be written in the form:

$$
V_{y}(z, t)=V_{p o}\left(g_{A}(z) \sin \omega t-g_{B}(z) \cos \omega \cdot t\right)
$$

As discussed earlier, the boundary condition on $V y$ at $z_{1}$ is $V / y\left(z_{1}=\right.$ $100 \mathrm{~km})=0$. For the boundary condition on $V / y$ at $z_{2}$, we take $V_{y}\left(z_{2}\right)=V / y$ $\left(\mu=0, z_{2}\right)$, where $V_{y}\left(\mu=0, z_{2}\right)$ is the solution to the force equation

$$
\rho\left(\alpha V_{y} / \partial t\right) \hat{y}=\left(y \times B_{0} / c\right) \hat{y}=\left(\sigma_{p} B_{0} / c\right)\left[E_{x_{0}}-V_{y} B_{0} / c\right] \hat{y}
$$

at $z=z_{2}$. We use this equation, in which viscous forces are neglected, to determine the upper boundary condition because by the definition of $z_{2}$ viscous forces are unimportant for $z \geq z_{2}$. As the boundary condition on $b_{y}$, we take $b_{y}\left(z_{1}=100 \mathrm{~km}\right)=0$. 
There are several approaches to the solution of (11) when

$\mu$, op and $\rho$ are general functions of $z$. It can be transformed to an integral equation, or the functions $g_{A}, g_{B}$ can be found by means of a variational principle, or a perturbation technique may be used. These methods are discussed in Appendix 2.

With $\sigma_{p}$ and $\mu$ constants and with $\rho=\rho_{0} E \times P(-z / H)$ where $\rho_{0}$ and $H$ are constants, (11) has a solution in terms of Bessel functions (Appendix 2).

Once $g_{A}$ and $g_{B}$ are known, $f_{y}$ and thus $S(z, t)$ follow from (9). We obtain a general expression for the coefficient $C$ in terms of integrals over $g_{A}, g_{B}$ by substituting (8) and (12) into (9) and integrating. The result is

where

$$
\begin{aligned}
S\left(z_{2}, t\right) & =-B_{0} b_{y}\left(z_{2}, t\right) / 4 \pi \\
& =V_{p o}\left(C_{A} \sin \omega t+C_{B} \cos \omega t\right) \\
& =V_{p o} C \sin (\omega t+\theta)
\end{aligned}
$$

and

$$
C_{A}=B_{0}^{2} / c^{2} \int_{z_{1}}^{z_{2}} \sigma_{p}\left(z^{\prime}\right)\left[1-g_{A}\left(z^{\prime}\right)\right] d z^{\prime}
$$

$$
\begin{aligned}
& C_{B}=B_{0}^{2} / C^{2} \int_{z_{1}}^{z_{2}} \sigma_{p}\left(z^{\prime}\right) g_{B}\left(z^{\prime}\right) d z^{\prime} \text {. Here } \\
& \theta=\tan ^{-1}\left(C_{B} / C_{A}\right) \text {, and }{ }^{z_{1}} C=\left(C_{A}^{2}+C_{B}^{2}\right)^{\frac{1}{2}}
\end{aligned}
$$

is the desired expression for the drag coefficient in terms of general functions $g_{8}$

The total rate of dissipation, viscous plus joule, in a column of unit cross section extending from $z_{1}$ to $z_{2}$ is the average of $S\left(z_{2}\right)$ Vpy $\left(z_{2}\right)$ over a cycle, which is $\left[. S\left(z_{2}\right) V_{p y}\left(z_{2}\right)\right] A V=\frac{1}{2} C \cos \theta V_{p o}^{2}$ 
We will examine three cases: the limiting case of $W=0$, the maximum frequency $w=0.6 \times 10^{-3} \mathrm{sec}^{-1}$, and the frequency $w=0.7 \times 10^{-4}$ $\sec ^{-1}$. In each case the role of viscosity is assessed. The $w=0$ limit serves to reveal, in a simple way, much of the underlying physics. For $W=0.6 \times 10^{-3} \mathrm{sec}^{-1}$, inertia is important and its effect on the drag coefficient is illustrated. These considerations lead to values of $C$ and $\theta$ from which the ionospheric dissipation rate is estimated.

\section{Discussion}

A. $w=0$

For $\quad w=0$ we take $z_{1}=80 \mathrm{~km}$ with $\quad V_{y}(z \leq 80 \mathrm{~km})=0$ and $\vec{E}=E_{x \circ} \hat{x} \quad=$ constant in space and time down to $80 \mathrm{~km}$. (The $z$ component of the electric field is still fairly small as far down as $80 \mathrm{~km}$ so we ignore it.) The $w=0$ problem with $z_{1}=80 \mathrm{~km}$ is physically equivalent to a time dependent situation in which than

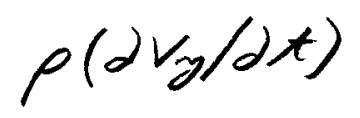<smiles>C=CC=CC=CC=CC</smiles>
is much greater for $z>80 \mathrm{~km}$, with so large for $z \leq 80 \mathrm{~km}$ that $\quad V_{y}\left(z \leq z_{2}\right)=0$. We take $V_{p y}\left(z \geq z_{2}\right)=V_{p o}$ ; then (8) becomes for this time independent case:

$$
E_{\times 0}=V_{p 0} B_{0} / c=\text { constant, } z \geq 80 \mathrm{~km} .
$$

Equations (9), (10), and (14) lead to

$$
\partial b_{y} / \partial z=-B_{0}\left(4 \pi \sigma_{p} / c^{2}\right)\left[V_{p_{0}}-V_{y}\right]
$$




$$
T=-B_{0} b_{y} / 4 \pi+\mu(\partial V / \partial / \partial z)=\text { constant }
$$

where $T$ is the total $y z$ stress. At the level $z_{2}$ we have $T \approx S\left(z_{2}\right)$. $=-B_{0} b_{y} / 4 \pi$ to good approximation. As one moves from $z_{2}$ downward, $\mid b y /$ decreases so that less of the stress is borne by the magnetic field and more by the velocity shear. Finally at $z_{1}=80 \mathrm{~km}, b_{y}=0$ and all the stress is viscous shearing stress.

We first consider the solutions to (15) when $\sigma_{p}=\sigma_{p o}=$ constant and $\mu=\mu_{0}=$ constant. These solutions illustrate what happens when the conductivity is increased. They are, for $z_{1} \leq z \leq z_{2}$ :

$$
V_{y}(z)=V_{p 0}\left[1-\frac{\sinh \left[\left(z_{2}-z\right) / D_{T}\right]}{\sinh \left[\left(z_{2}-z_{1}\right) / D_{T}\right]}\right]
$$

$$
\begin{aligned}
b_{y}(z)= & -V_{p 0} 4 \pi\left(\mu_{0} \sigma_{p 0} / c^{2}\right)^{\frac{1}{2}} \operatorname{coth}\left[\left(z_{2}-z_{1}\right) / D_{T}\right] \\
& x\left[1-\frac{\operatorname{coch}\left[\left(z_{2}-z\right) / D_{T}\right]}{\cos h\left[\left(z_{2}-z_{1}\right) / D_{T}\right]}\right], \\
D_{T}= & \left(\mu_{0} c^{2} / B_{0}^{2} \sigma_{p_{0}}\right)^{\frac{1}{2}} \quad \text { is the transfer distance, }
\end{aligned}
$$

that is, the distance required for the transfer of stress from the magnetic field to viscous shear. Plots of these solutions are shown in Figs. 6, 7. From (16a) we have that

$$
\operatorname{Vg}\left(z \geq z_{1}+D_{T}\right) \approx V_{p o} \text {. Thus when } D_{T} \text { is small, }
$$
$V_{y}(z) \approx V_{\rho o}$ down to a lower level, and the magnetic field moves with the medium down to that level. Further, when $D_{T}$ is small, we see from Fig. 6 
that the velocity shear at $z_{1}$ is increased, and thus

$$
T=\mu_{0}\left(\partial V_{y} / \partial z\right)\left(z_{1}\right)
$$

$\approx S\left(z_{2}\right)$ is increased. Therefore when

the field is frozen into a lower level, and

In Fig. 6 the distance between the

\%o is increased ( $\mu_{0}$ fixed), $S\left(z_{2}\right)$ is increased.

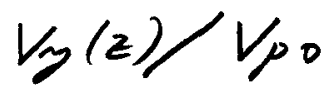

$=1$ line

and the $V_{y}(z) / V_{p o}$
$E_{x_{0}}-V_{y} B_{0} / c$

From (16b), the drag coefficient is readily found. We obtain

$$
\begin{array}{r}
c=B_{0}\left(\mu_{0} \sigma_{\rho 0} / c^{2}\right)^{\frac{1}{2}}\left(\operatorname{coth}\left[\left(z_{2}-z_{1}\right) / D_{T}\right]\right) \\
x\left(1-1 / \cosh \left[\left(z_{2}-z_{1}\right) / D_{T}\right]\right)
\end{array}
$$

Now $z_{2}$ must be such that $\left(z_{2}-z_{1}\right) / D_{T} \quad \gg>1$; otherwise, there would be significant viscous dissipation above $z_{2}$. It follows that $c=B_{0}\left(\mu_{0} \sigma_{p 0} / c^{2}\right)^{\frac{1}{2}}$ to good approximation. For the $\mu_{0}$ and $\sigma_{p_{0}}$ values of Figs. 6, 7, C is found to be:

$$
\begin{array}{ll}
\sigma_{p 0}=1 \times 10^{-17} \text { EMU } & C=2.7 \times 10^{-11}\left(\text { dynes-sec } / \mathrm{cm}^{3}\right) \\
\sigma_{p 0}=1 \times 10^{-16} \text { EMU } & C=8.7 \times 10^{-11}\left(\text { dynes-sec } / \mathrm{cm}^{3}\right) \\
\sigma_{p 0}=3 \times 10^{-15} \text { EMU } & C=47.0 \times 10^{-11}\left(\text { dynes-sec } / \mathrm{cm}^{3}\right)
\end{array}
$$

Values of the drag coefficient were also found for non-constant $\mu$ and $\sigma_{p}$. Fig. 8 shows various profiles of the Pederson conductivity $\sigma_{p}$. Fig. 9 shows four approximate representations of the curves of Fig. 8. These approximate curves differ only by a scale factor. Using the $\sigma_{p}(z)$ profiles of Fig. 9 and with an approximate analytic expression for $\mu(z)$ (see Appendix 1), the equations (15) were numerically integrated from $z_{1}=80 \mathrm{~km}$ to $z_{2}=300 \mathrm{~km}$. The values of $C$ thus found are: 


$$
\sigma_{p}(100 \mathrm{~km})(E M U) \quad C\left(\text { dynes-sec } / \mathrm{cm}^{3}\right)
$$

Sunspot maximum: day $4.5 \times 10^{-16} \quad 5.02 \times 10^{-11}$

$$
\text { night } \quad 5.4 \times 10^{-18} \quad 1.52 \times 10^{-11}
$$

Sunspot minimum: day

$$
2.5 \times 10^{-16} \quad 4.64 \times 10^{-11}
$$

$$
\text { night } \quad 5.4 \times 10^{-17} \quad 3.79 \times 10^{-11}
$$

Again, $C$ increases for increasing $\sigma_{\rho}$, although $C$ is not so sensitive to $\sigma_{\bar{P}}$ as in the constant parameter case. This is also the situation when $w>0$. In this $w=0$ case the dissipation per unit area is $C V_{p}^{2}$. From the numerical solutions to (15) for non-constant $\sigma_{p}$ (Fig. 9) and (Appendix 1), it is found that as $\sigma_{p}$ increases, the viscous dissipation is an increasingly larger fraction of the total dissipation. For example, viscous dissipation is found to be about three times larger than joule dissipation for night-time, sunspot maximum and about ten times larger than joule dissipation for daytime, sunspot maximum. B. $w=0.6 \times 10^{-3} \sec ^{-1}$

In this case $V_{y}$ is time dependent so that the inertial term in the force equation (10) does not vanish. As in the $W=0$ problem, we first consider constant parameters, that is, we solve (11) for

$$
\begin{aligned}
& \sigma_{p}(z)=\sigma_{p_{0}}=\text { constant } \\
& \mu(z)=\mu_{0}=\text { constant } \\
& \rho(z)=\rho_{0} \exp \left[-\left(z-z_{0}\right) / H \quad\right]
\end{aligned}
$$

where $\rho_{0}, H$, and $z_{0}$ are constants. With these conditions an exact solution can be found in terms of Bessel functions (Appendix 2). When the quantity

$$
V=H / D_{T}=H /\left(\mu_{0} C^{2} / B_{0}^{2} \sigma_{p_{0}}\right)^{\frac{1}{2}}
$$
is an integer, this solution assumes a particularly simple form. For values of 
etc., appropriate to the dissipation region, $\sqrt{ }$ lies in the range $0<\square \leq 3$. We will take $\quad \mathscr{V}=1$ with

$$
\begin{aligned}
\sigma_{p o}(E S U) / C^{2} & =\sigma_{p o}(E M U) \quad=1.2 \times 10^{-15} \text { EMU } \\
\mu_{0} & =3 \times 10^{-4} \mathrm{~g} / \mathrm{cm}-\mathrm{sec} \\
H & =10 \mathrm{~km}, \quad \rho_{0}=2.8 \times 10^{-10} \mathrm{~g} / \mathrm{cm}^{3}, \quad Z_{0}=100 \mathrm{~km}
\end{aligned}
$$

The viscosity is relatively constant through the dissipation region with $3 \times 10^{-4} \mathrm{~g} / \mathrm{cm}-\mathrm{sec}$ being typical. For values of $\sigma_{p} \circ$ and $H$ appropriate to that region, viscous forces are more significant when $V=1$ than when $v=2$ or $V=3$. We have taken $\quad V=1$ in order that the effect of viscosity may be more pronounced. The function (z) defined by these parameters is show $n$ in Fig. 10, along with points of the density curves for sunspot maximum and minimum. For the parameters (17), the solutions $g_{A}(z), g_{B}(z)$ to (11) are plotted in Figs. 11, 12 (see Appendix 2). In this time dependent case, momentum transferred into the dissipation region by magnetic stressing produces $V / y$ and is further transferred by viscous stresses. For $w=0$ and for the conductivity and viscosity values of (17), the solution (z) is shown in Fig. 11. Below $140 \mathrm{~km}$, 9 is much greater than $g_{A}$ or $g_{B}$. This is because the density at lower levels is so great that little velocity is acquired by the time the driving force $\left(y \times B_{0} / C\right) \hat{y}$ is reversed. The effect of inertia is thus to decrease $\mid V / \quad$ significantly at lower levels and thereby to increase the drag coefficient $C$ (see Eqn. 9). it is of interest to examine the solution $V y(z, t)$ when $\mu=0$. With $\mu=0$ and for $v_{p}$ and $\rho$ general functions of $z,(11)$ has the solution, which we denote by a superbar, 
the approximate solutions $\bar{g}_{A}, \bar{g}_{B}$ and $\tilde{g}_{A}, \tilde{g}_{B}$, the quantities $C$ and $\theta$ may be found by (13). From $g_{A}, g_{B}$ we obtain:

$$
C=2.01 \times 10^{-9} \text { dynes-sec } / \mathrm{cm}^{3}, \quad \theta=0.24 \text { radians; }
$$

from $\bar{g}_{A}, \bar{g}_{B}$ we obtain:

$$
\bar{C}=1.97 \times 10^{-9} \text { dynes-sec } / \mathrm{cm}^{3}, \quad \bar{\theta}=0.25 \text { radians; }
$$

and from $\widetilde{g}_{A}, \widetilde{g}_{B}$ we obtain:

$$
\widetilde{C}=2.02 \times 10^{-9} \text { dynes-sec } / \mathrm{cm}^{3}, \quad \tilde{\theta}=0.14 \text { radians. }
$$

Thus $\tilde{g}_{A}, \widetilde{g}_{B}$ give an accurate value of $C$. The value of the drag coefficient associated with the $g(z)$ curve in Fig. 11 is $C=0.3 \times 10^{-9}$ dynes-sec/ $\mathrm{cm}^{3}$.

We note that the dissipation $\frac{1}{2} C \cos \theta V_{p o}^{2} \quad$ is larger than $\frac{1}{2} \bar{C} \cos \bar{\theta} V_{p o}^{2} \quad$ because in the former case work must be done against viscous forces.

To study the effect on the drag coefficient of varying $\sigma_{p o}$ and $H$ we have found $\widetilde{C}$ and $\tilde{\theta}$ for four cases, shown in Table 1. ${ }^{9}$ The numbers in Table 1 may be compared with the values of $C$ found for the same parameters but with $w=0$. It is seen that $C$ is larger in the high frequency case. It is also seen from Table 1 that $C$ is almost directly proportional to $\sigma_{p^{0}}$
${ }^{9}$ In all but one of the cases of Table $1, z_{2}=300 \mathrm{~km}$. In case $2, z_{2}=475 \mathrm{~km}$. For case
we have required that $\widetilde{g}_{A}, \widetilde{g}_{B}$ satisfy at $300 \mathrm{~km}$ the boundary condition appropriate to $z_{2}=475 \mathrm{~km}$. This underestimates $\widetilde{C}$ and thus the inequality. For constant conductivity $\sigma_{p}(z)=\sigma_{p o}, C$ is given more accurately by $\widetilde{C}$ at low $\sigma_{p o}$ values (e.g., $\sigma_{p o}=1.0 \times 10^{-17} \mathrm{EMU}$ ) than by $\bar{C}$. Therefore, values of $\widetilde{C}$ are shown in Table 1. We expect that these $\widetilde{C}$ values are within $5 \%$ of $C$. 


$$
\begin{aligned}
& \bar{V}_{y}(z, t) \equiv V_{y}(z, t, \mu=0) \\
& =V_{p o}\left[\left\{V /\left(1+\omega^{2} T^{2}\right)\right\} \sin \omega t-\left\{\omega T /\left(1+\omega^{2} T^{2}\right)\right\} \cos \omega t\right] \\
& =V_{p o}\left[\bar{g}_{A}(z) \sin \omega t-\bar{g}_{B}(z) \cos \omega t\right]
\end{aligned}
$$

where $\tau=c^{2} \rho / B_{0}^{2} \sigma_{p}$

. When $\boldsymbol{T} T<1$,

we have $V_{y} \approx V_{p o}$ sinewt

, that is, the neutrals move

closely with the plasma. We note that for decreasing $w$ the amplitude

$$
\begin{aligned}
& \left|V_{y}(z, t)\right|=V_{p o}\left(\bar{g}_{A}^{2}+\bar{g}_{B}^{2}\right)^{\frac{1}{2}} \\
& V_{p o} /\left(1+w^{2} \tau^{2}\right)^{\frac{1}{2}} \quad \text { increases. }
\end{aligned}
$$

For the parameters (17), but with $\quad \mu_{0}=0, \bar{g}_{A}, \bar{g}_{B}$ are plotted in Figs. 11, 12. The bends are less sharp for the $g_{A}, g_{B}$ curves than for the curves $\bar{g}_{A}, \bar{g}_{B}$. This is just the effect viscosity should have. It is clear that for the $g_{A}, g_{B}$ curves of Figs. 11, 12, inertia is much more important than viscosity.

An approximate solution to (11) can also be found by means of a variational principle (Appendix 2). This is done by expanding $g_{A}, g_{B}$ in a Fourier series and truncating the series after two terms. The approximate solutions thus found are denoted by $\widetilde{g}_{A}, \widetilde{g}_{B}$. For the parameter values (17), these solutions are plotted in Figs. 11, 12. They are as close as can be expected with a two-term Fourier series to the $g_{A}, g_{B}$ curves. Clearly in the case of Figs. 11,12 $\bar{g}_{A}, \bar{g}_{B}$ are better approximations to $g_{A}, g_{B}$ than are
$\widetilde{g}_{B}$. From the exact solutions including viscosity $g_{A}, g_{B}$ and from 
$-27-$

This is because, for $w=0.6 \times 10^{-3} \mathrm{sec}^{-1}, / V_{y} / / / V_{p o l}$ is small (see Eqn。(9)). The larger value of $H$ corresponds to larger mass densities (Fig. 10).

Thus far, the functions $\sigma_{p}, \mu$, and $H$ have been taken to be constant. Fig. 13 shows $\bar{g}_{A}$ and $\bar{g}_{B}$ (solid lines) found from the daytime, sunspot maximum $\sigma_{\rho}$ (Fig. 8) and the sunspot maximum $\rho$ (Fig. 10). We consider daytime, sunspot maximum conditions, for which $\sigma_{p}$ is largest, in order to obtain maximum values of the drag coefficient and the dissipation rate. The solutions $g_{A}, g_{B}$ including viscosity, lie within the dashed curves of Fig. 13. The dashed curves were found as follows: The viscous force was taken as the larger of $\mu\left[\left(\partial \bar{g}_{A} / \partial z\right)\left(z_{B}\right)-\left(\partial \bar{g}_{A} / \partial z\right)\left(z_{\alpha}\right)\right], \mu\left[\left(\partial \bar{g}_{B} / \partial z\right)\left(z_{B}\right)-\left(\partial \bar{g}_{B} / \partial z\right)\left(z_{\alpha}\right)\right]$ with $\bar{g}_{A}, \bar{g}_{B}$ from Fig. 13 and with $\left(z_{\beta}-z_{\alpha}\right) \approx D_{V}\left(z_{\alpha}\right)$ (where $D_{v}=(\mu(z) / \rho(z) \omega)^{\frac{1}{2}} \quad$ ). The maximum amount $\Delta g$ by which the viscous force can alter $\bar{g}_{A}, \bar{g}_{B}$ was obtained by equating $m(\Delta g / \Delta t)_{Z_{B}}$ to the viscous force and solving for $\Delta g$.

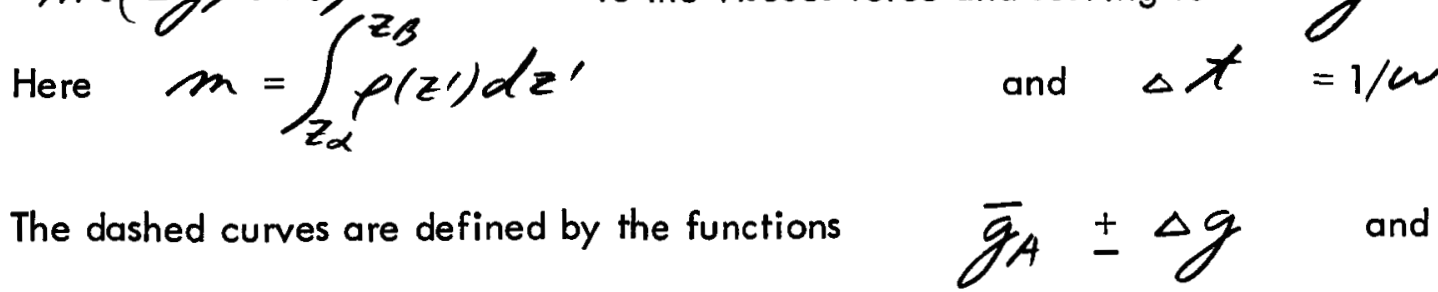
$\bar{g}_{B} \pm \Delta g$. By finding $\Delta g$ in this manner, the effect of viscosity on the motion is overestimated. Thus the curves $g_{A}, g_{B}$ lie within the dashed curves. ( $\Delta g$ was calculated from the $\bar{g}_{A}, \bar{g}_{B}$ curves of Figs. 11,12 , and it was found that $g_{A}$, at most levels, was closer to $\bar{g}_{A}$ than to either $\bar{g}_{A}+\Delta g$ or $\bar{g}_{A}-\Delta g$, and similarly for $g_{B}$, $\bar{g}_{B}$. Also, $g_{A}$ and $g_{B}$ were everywhere within $\pm \Delta g$ of 
$-28-$

$\bar{g}_{A}$ and $\bar{g}_{B}$.) From the $\bar{g}_{A} \pm \Delta g, \bar{g}_{B} \pm \Delta g$ profiles
of Fig. 13, the quantities $\bar{C} \pm \Delta C, \bar{\theta} \pm \Delta \theta \quad$ can be found by doing the integrals in (13) numerically. We thus obtain

$$
\begin{array}{ll}
\bar{C} \pm \Delta C & =(4.2 \pm 0.5) \times 10^{-9} \text { dynes -sec } / \mathrm{cm}^{3} \\
\bar{\theta} \pm \Delta \theta & =(0.29 \pm 0.10) \text { radians. }
\end{array}
$$

Now the total dissipation including viscosity $\frac{1}{2} C \cos \theta V_{p o}^{2}$ must be greater than $\frac{1}{2} \bar{C} \cos \bar{\theta} V_{p o}^{2}$

- It is reasonable to assume that in general $C$ will be greater than $\bar{C}$ and $\theta$ less than

$\bar{\theta}$, as was the case for the solutions of Figs. 11, 12. Then $C$ will lie in the range $\left(\bar{C}=4.2 \times 10^{-9}\right.$ dynes-sec $\left./ \mathrm{cm}^{3}\right) \leq C \leq(\bar{C}+\Delta C=$ $4.7 \times 10^{-9}$ dynes-sec $/ \mathrm{cm}^{3}$ ), and $\theta$ in the range $(\bar{\theta}-\Delta \theta=0.19$ radians) $\leq \theta \leq(\theta=0.29$ radians). For the same conditions but $\omega=0$ it was found that $\quad C=5.02 \times 10^{-11}$ dynes -sec $/ \mathrm{cm}^{3}$. Clearly the effect of inertia, in diminishing $\mid V y /$ and hence increasing $C$, is a significant one. The quantity $C \cos \theta \quad$ will lie in the range $(\bar{C} \cos \bar{\theta}=$ $4.0 \times 10^{-9}$ dynes-sec $\left./ \mathrm{cm}^{3}\right) \leq C \cos \theta \leq([\bar{C}+\Delta C$ ] cos $[\bar{\theta}-\Delta \theta \quad]=4.6 \times 10^{-9}$ dynes -sec $/ \mathrm{cm}^{3}$ ).

Now the average rate of viscous dissipation per unit area is

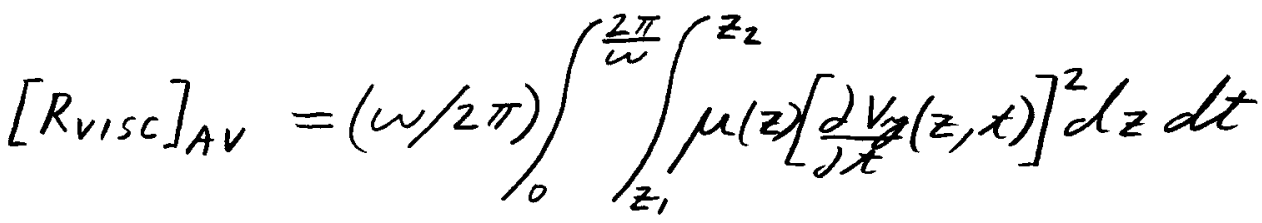

$$
\begin{aligned}
& =\frac{1}{2} v_{p 0}^{2} \int_{z_{1}}^{z_{2}} \mu(z)\left[\left(\partial g_{A} / \partial_{z}\right)^{2}+\left(\partial g_{B} / \partial_{z}\right)^{2}\right] d z
\end{aligned}
$$


$-29-$

Because the functions $g_{A}, g_{B}$ are always smoother functions of $z$ than are $\bar{g}_{A}, \bar{g}_{B}$, it follows that

$$
\left[R_{V I S C}\right]_{A V}<\left[\overline{R_{V I S C}}\right]_{A V} \equiv \frac{1}{2} V_{p O}^{2} \int_{z_{1}}^{z_{z}} \mu(z)\left[(\partial \bar{g} A \partial z)^{2}+\left(\partial \bar{g}_{\Delta} / \partial z\right)^{2}\right] d z \text {. }
$$

From the functions $\bar{g}_{A}, \bar{g}_{B}$ of Fig. 13 we have evaluated $\left[\overline{R_{V I S C}}\right]_{A V} / V_{\rho 0}^{2}$. We find that $\left[\overline{R_{V I S C}}\right]_{A V} /$ $\frac{1}{2} \bar{C} \cos \bar{\theta} V_{\rho o}^{2}=.014$. That is, for $u=0.6 \times 10^{-3} \sec ^{-1}$ and daytime, sunspot maximum conditions, viscous dissipation accounts for less than $1.4 \%$ of the total dissipation. For conditions other than daytime, sunspot maximum, the upper limit on the viscous dissipation is somewhat higher, ranging up to about $15 \%$ of the total dissipation (for night, sunspot maximum conditions). Therefore, this $W=0.6 \times 10^{-3} \mathrm{sec}^{-1}$ case is opposite to that of $W=0$, where viscous dissipation was primary. Whereas for $w=0$ there was no inertia and viscous forces were important, for $W=0.6 \times 10^{-3} \mathrm{sec}^{-1}$ the inertial term in the force equation is dominant over the viscous term.

C. $\quad w=0.7 \times 10^{-4} \mathrm{sec}^{-1}$

The frequency $\quad W=0.7 \times 10^{-4} \mathrm{sec}^{-1}$ is the characteristic frequency for motions over the central polar regions $\left(A_{p} D\right.$ in Fig. 2). For this frequency, visecosily is more and inertia less significant than for $\omega=0.6 \times 10^{-3} \sec ^{-1}$. Nevertheless, the $\mu=0$ approximation is of useful accuracy in this case. Proceding as before, we find that for daytime, sunspot maximum conditions $1.6 \times 10^{-9}$

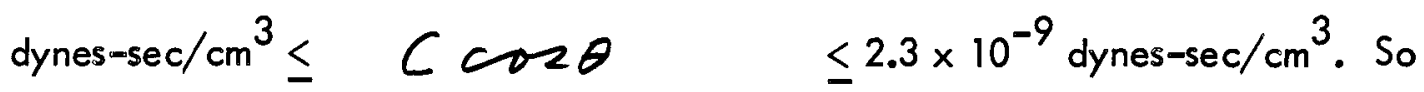
$\cos \theta$ is not much smaller in this case than for $\omega=$ 
$0.6 \times 10^{-3} \sec ^{-1}$

As mentioned earlier, Hall current and Coriolis forces will modify and thus alter the joule and viscous dissipations. We have made estimates of these effects and found that in this lower frequency case they lead to an increase in

$$
\cos \theta \quad \text { by perhaps as much as } 50 \text { percent }_{g} \text { (these effects being }
$$
negligible for $\quad W=0.6 \times 10^{-3} \mathrm{sec}^{-1}$ ).

We find, for $W=0.7 \times 10^{-4} \sec ^{-1}$, that viscous dissipation is for all conditions less than 40 per cent of the total dissipation (and is less than 10 per cent for daytime, sunspot maximum conditions).

$$
\text { For } 0.7 \times 10^{-4} \mathrm{sec}^{-1} \leq \quad w \leq 0.6 \times 10^{-3} \mathrm{sec}^{-1} \text {, and assuming }
$$

the conductivities and mass densities of Figs. 8,10 , we have found that (in the polar ionosphere) viscous dissipation, $[R \text { VisC }]_{A V}$, is always less than 40 per cent of the total dissipation $\frac{1}{2} C \cos \theta V_{p o}^{2}$ The fraction 40 per cent occurs for $\omega=0.7 \times 10^{-3} \sec ^{-1}$ and nighttime, sunspot maximum conditions, for which $\theta_{p}$ is quite small at low levels. As $w$ increases, the viscous dissipation rate decreases and the rate of joule dissipation increases, so that, for $W=0.6 \times 10^{-3} \mathrm{sec}^{-1}$ and nighttime, sunspot maximum conditions, viscous dissipation is less than 15 per cent of the total. With increasing $\sigma_{p}$, the joule dissipation rate increases. Consequently, for $W=0.6 \times 10^{-3} \mathrm{sec}^{-1}$ and daytime $e_{0}$ sunspot maximum conditions, viscous dissipation accounts for less than 1.4 persent of the total. Thus joule dissipation is always dominant over viscous dissipation. 
The integrated joule dissipation rate,

$$
\int_{z_{1}}^{z_{2}} \sigma_{p}\left(E_{x 0}-v_{y} B_{0} / c\right)^{2} d z
$$

will not be much different from

$$
R_{j}^{\prime}=\int_{z_{1}}^{z_{2}} \sigma_{p}\left(E_{x_{0}}\right)^{2} d z
$$

provided

$$
\left|V_{y} B_{0} / E_{x 0} c\right|^{z_{1}}=\left|V_{y} /\right| V_{p o} \mid
$$

is small in the height range where $\sigma_{p}$ is largest. Because of this, for $w=$ $0.6 \times 10^{-3} \sec ^{-1}$ and daytime, sunspot maximum conditions $R_{y}^{\prime}$ is only about 20 per cent larger than $R_{j}$. But for $w=0.7 \times 10^{-4} \mathrm{sec}^{-1}$ and nighttime sunspot maximum conditions, the above situation is not too well achieved, and $R_{j}^{\prime}$ is about three times larger than $R_{j}$. All other cases lie between these extremes. Hence, in obtaining the joule dissipation rate the neutral gas velocity can, to rough approximation, be neglected altogether.

From the values of $C$ and $\theta$ found above, it is easy to find the rate at which energy is dissipated in the polar ionospheres. For the high velocity regions $E, F$ and $G, H$ in Fig. 3, the plasma velocity is $V_{p o} \approx$ $1 \mathrm{~km} / \mathrm{sec}$. These two regions each have a length of about $5 \times 10^{3} \mathrm{~km}$ and a width of about $400 \mathrm{~km}$ for a total area, including both hemispheres, of .8 $\times 10^{17} \mathrm{~cm}^{2}$. The characteristic frequency appropriate to the regions $E, F$ and $G, H$ is $\quad W=$ $0.6 \times 10^{-3} \sec ^{-1}$. From above, we have that $\frac{1}{2} C \cos \theta \approx$ $2 \times 10^{-9}$ dynes-sec $/ \mathrm{cm}^{3}$. The dissipation rate for the regions $E, F$ and $G, H$ is then

$$
\phi=(\text { Area }) \times \frac{1}{2} C \cos \theta \times V_{p o}^{2} \approx 2 \times 10^{18}
$$

ergs/sec.

For the central polar region in Fig. 3: (Area) $\approx 2 \times 10^{17} \mathrm{~cm}^{2}$, $w=0.7 \times 10^{-4} \sec ^{-1}, \quad \frac{1}{2} C \cos \theta \approx 1 \times 10^{-9}$ dynes-

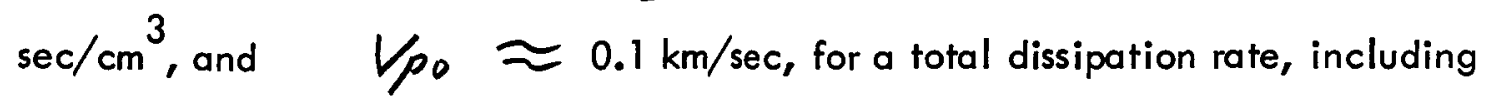


both hemispheres, of $4 \times 10^{16} \mathrm{ergs} / \mathrm{sec}$, which is negligible in comparison with

Then for convective velocities of $1 \mathrm{~km} / \mathrm{sec}$, viscosity and electrical resistivity result in the dissipation of energy at the rate $\phi \approx 2 \times 10^{18} \mathrm{ergs} / \mathrm{sec}$, assuming daytime, sunspot maximum conditions. ${ }^{10}$ Since this dissipation occurs in the high velocity regions where the characteristic frequency is $\omega=0.6 \times 10^{-3}$ $\sec ^{-1}$, viscous dissipation is at most 15 percent of the total ionospheric dissipation, The above value of $\varnothing$ was obtained for daytime, sunspot maximum conditions for which $\sigma_{p}$, and thus $C \cos \theta$, is largest. Now we have used mid-latitude conductivities which, according to Chapman (1956), are larger than polar conductivities. Thus the value $2 \times 10^{18}$ ergs/sec is perhaps an overestimate, being based on $V_{p}$ values which are possibly somewhat high. On the other hand, auroral corpuscular bombardment may lead to significantly enhanced conductivities. It appears (Bostrom, 1964) that these enhanced conductivities are not much greater than the daytime, sunspot maximum $\sigma_{p}$ we have considered. Hence $2 \times 10^{18} \mathrm{ergs} / \mathrm{sec}$ is a reasonable upper bound for the ionospheric joule and viscous dissipation rate.

We conclude that, if some mechanism can provide energy at least at the rate of $2 \times 10^{18} \mathrm{erg} / \mathrm{sec}$, then the convection can be maintained. We ask whether the solar wind might possible provide this much energy? About $10 \mathrm{~km} / \mathrm{sec}$ is a reasonable value for the convection velocity of the outer magnetospheric plasma. The solar

${ }^{10}$ Axford and Hines (1961) estimated $\phi$ to be of the order of $10^{18} \mathrm{ergs} / \mathrm{sec}$ and Axford (1963) obtained $10^{17}$ ergs/sec for the joule dissipation, with an uncertainty of a factor of 10. 
wind dynamic pressure, at active times, is typically $\quad S_{5 \mathrm{~W}}=3 \times 10^{-7} \mathrm{dynes} / \mathrm{cm}^{2}$ (20 atoms $/ \mathrm{cm}^{3}, 10^{3} \mathrm{~km} / \mathrm{sec}$ ). This stress is applied to the magnetopause over a frontal area of $A_{M}=3 \times 10^{20} \mathrm{~cm}^{2}$ so that an upper limit on the rate at which the solar wind could do work would be $\phi_{5 \mathrm{w}}=5_{5 \mathrm{w}} \times(10 \mathrm{~km} / \mathrm{sec}) \times \mathrm{Am}=$ $1 \times 10^{20}$ ergs/sec. This is fifty times larger than the energy consumed by the joule and viscous dissipation, $\varnothing$. In addition to joule and viscous dissipation, energy can be dissipated by ring current decay and by the collisional ionization processes associated with aurorae. The dissipation associated with ring current decay and the aurora can amount altogether to perhaps as much as $7 \times 10^{18} \mathrm{ergs} / \mathrm{sec}$ for convective velocities. of $1 \mathrm{~km} / \mathrm{sec}$ (Axford, 1963). Hence, joule plus viscous dissipation is not necessarily dominant over these other types of dissipation. The total dissipation will in any case be less than $1 \times 10^{19}$ ergs/sec. Thus so far as the energetics are concerned, it is feasible that the solar wind drive the convection. But even though this may be energetically feasible, it cannot be said that the solar wind does, in fact, drive the convection because the effective cross section over which it does work on the magnetosphere is not known. However, it can be said that if the effective cross section of the magnetosphere is $1 / 10 \mathrm{AM}$, the solar wind can drive the convection at velocities of about $1 \mathrm{~km} / \mathrm{sec}$. On the basis of this convection, various geophysical phenomena, such as the DS current system and particle energization, can be understood, as was pointed out by Axford and Hines.

Acknowledgments: I am greatly indebted to Professor E. N. Parker for suggesting this problem, for many helpful discussions of it, and for guidance toward its solution. I also wish to express my thanks to Professor C. O. Hines for a number of useful discussions. 


\section{APPENDIX 1}

We have found the stress tensor for an ion-neutral gas in the presence of a magnetic field. The Chapman-Cowling method was used (Chapman and Cowling, 1960). Ion-ion collisions were considered out to the Debye length. The neutralneutral and neutral-ion interactions were treated as hard sphere interactions. These results will be published later. It was found that in the lower ionosphere the total ion-neutral viscosity is essentially the neutral gas viscosity and thus not dependent on $\vec{B}$. The viscous stress tensor is then

$$
R_{i j}=\mu\left[\partial V_{i} / \partial x_{j}+\partial V_{j} / \partial x_{i}-(2 / 3) \delta_{i j}(\partial V k / \partial x k)\right] \text {. }
$$

The viscosity $\mu$ was evaluated for ionospheric levels. It was found that in the range $80 \mathrm{~km}$ to $350 \mathrm{~km} \mu$ can be represented to within $20 \%$ by the function

$$
\mu(z)(\mathrm{g} / \mathrm{cm}-\mathrm{sec})=2.42 \times 10^{-6} \times Z(\mathrm{~km})-6.9 \times 10^{-5} .
$$


$-35-$

APPENDIX 2

Substituting (12) into (11) yields the pair of equations

$$
\frac{\partial}{\partial z}\left(\mu \frac{\partial g_{A}}{\partial z}\right)-\frac{B_{0}^{2}}{4 \pi \lambda p} g_{A}-\rho \omega g_{B}=-\frac{B_{0}^{2}}{4 \pi \lambda_{p}}
$$

(Ala)

$$
\frac{\partial}{\partial z}\left(\mu \frac{\partial g_{B}}{\partial z}\right)-\frac{B_{0}^{2}}{4 \pi \lambda_{\rho}} g_{B}+\rho w g_{A}=0
$$

$\left(\lambda_{p}=c^{2} /\left(4 \pi \sigma_{p}\right)\right) \quad . \quad$ These are iv

$$
\begin{gathered}
I=\left[-\frac{1}{2} \mu\left(\frac{\left.\partial g_{\theta}\right)^{2}}{\partial \partial_{z}}+\frac{1}{2} \mu\left(\frac{\left.\partial g_{B}\right)^{2}}{\partial \partial_{z}}-\frac{B_{0}^{2}}{8 \pi \lambda_{p}}\left(g_{A}^{2}-g_{B}^{2}\right)\right.\right.\right. \\
\left.-\rho \omega g_{A} g_{B}+\frac{B_{0}^{2}}{4 \pi \lambda_{p}} g_{A}\right] .
\end{gathered}
$$

where

The approximate solutions $\widetilde{g}_{A}, \widetilde{g}_{B}$ were found by letting

$$
\begin{aligned}
& \widetilde{g}_{A}=\frac{1}{2}(1-\cos g z)+a_{1} \sin q z+a_{2} \sin 2 g z \\
& \tilde{g}_{B}=b_{1} \sin q z+b_{2} \sin 2 q z
\end{aligned}
$$

and then choosing $a_{1}, a_{2}, b_{1}, b_{2}$ to give an extremum of $M$. Here $q=\pi /\left(z_{2}-z_{1}\right)$ and the term $\frac{1}{2}(1-\cos g z) \quad$ is introduced to satisfy boundary conditions. 
For

$$
\begin{aligned}
& \mu=\mu_{0}=\text { constant } \\
& \sigma_{p}=\sigma_{p 0}=\text { constant } \\
& \rho=\rho_{0} e^{\frac{z}{H}} \text { with } \rho_{0}, H \text { constants, }
\end{aligned}
$$

(11) reduces to

$$
\begin{aligned}
& \frac{\partial^{2} V_{y}}{\partial z^{2}}-\frac{1}{D_{T}^{2}} V_{y}-\frac{1}{D_{V}^{2}} \operatorname{ExP}\left(-\frac{z}{H}\right) \frac{1}{\omega} \frac{\partial V_{y}}{\partial t}=-\frac{V_{p 0}}{D_{T}^{2}} \sin \omega t \\
& \left(D_{v}=\sqrt{\mu_{0} / \rho_{0} w}\right) \text {. }
\end{aligned}
$$

Transforming to the new variable $\quad \xi=\operatorname{ExP}\left(-\frac{z}{H}\right) \quad$ (A2) becomes

$\xi^{2} \frac{\partial^{2} V_{y}}{\partial \xi^{2}}+\xi \frac{\partial V_{y}}{\partial \xi}-v^{2} V_{y}-\frac{\epsilon^{2}}{\omega} \xi \frac{\partial V_{y}}{\partial t}=-v^{2} V_{p} \cdot \sin m t$

where $v^{2}=H^{2} / D_{T}^{2} \quad$ and $\epsilon^{2}=H^{2} / D_{v}^{2}$

We take $V y$ to vary as $\operatorname{EXP}(i$ int) and consider the homogeneous equation associated with (A3), viz.,

$$
\xi^{2} \frac{\partial^{2} V_{y}}{\partial \xi^{2}}+\xi \frac{\partial V_{y}}{\partial \xi}-v^{2} V_{y}-i \epsilon^{2} \xi V_{y}=0
$$

This equation has solutions of the form $Z_{2 v}\left(2 \operatorname{ExP}\left(i \frac{3 \pi}{4}\right) \frac{H}{D_{v}} \xi^{\frac{1}{2}}\right)$ where $Z_{2 v} \quad$ is any of the solutions to Bessel's equation of order 2 v. The particular solution to (A3) can be expressed in terms of Bessel functions times integrals of Bessel functions. Thus expressed, it reduces to infinite sums of Bessel functions over the order. These sums are difficult to evaluate and so we have found other forms of the particular solution. When $V$ is not an integer, the particular 
solutions to $(A 3)$ are:

$$
\begin{aligned}
& g_{A}(\text { PART. })=\sum_{n=0}^{\infty} \frac{(-1)^{n} \epsilon^{4 n}}{\left([2 m]^{2}-v^{2}\right)\left([2 m-1]^{2}-v^{2}\right) \cdots \cdot\left(1-v^{2}\right)} \xi^{2 n} \\
& g_{B(\text { PART })}=\sum_{n=0}^{\infty} \frac{(-1)^{n-1} \epsilon^{4 n-2}}{\left([2 m-1]^{2}-v^{2}\right)\left([2 m-2]^{2}-v^{2}\right) \cdots \cdots\left(1-v^{2}\right)} \xi^{2 n-1}
\end{aligned}
$$

which are useful if $\epsilon$ is sufficiently small. When $v$ is an integer the particular solutions are polynomials in inverse powers of $\xi$ of which the simplest is that for $v=1$, viz.

$$
\begin{aligned}
& g_{A}(P A R T)=0 \\
& g_{B}(P A R T)=-\frac{1}{\epsilon^{2} \xi}
\end{aligned}
$$

Thus when $v$ is an integer the particular solutions assume a simple form. For integral $v$ the Bessel function solutions of the homogeneous equation (A4)

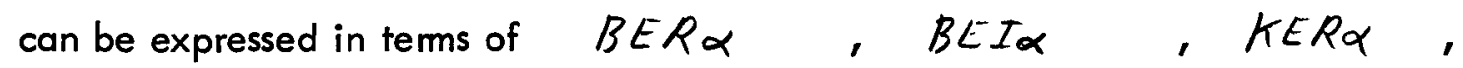
KEI $\alpha$ functions which are tabulated up to $\alpha=3$ (Young and Kirk, 1964; Tolke, 1936). The solutions $g_{A}, g_{B}$ shown in Figs. 11,12 were thus evaluated.

The differential equation (11), for general functions $\mu, \sigma_{p}$ and $\rho$, plus the boundary conditions $\quad a_{2}(t)=V_{y}\left(z_{2}, t\right)$ and $a_{1}(t)=\lg \left(z_{1}, t\right)$ is equivalent to the integral equation: 
$-38-$

$$
\begin{aligned}
V_{y}(z, t)= & \frac{1}{\left[M\left(z_{2}\right)-M\left(z_{1}\right)\right]} \\
x\{[M(z) & \left.-M\left(z_{1}\right)\right] a_{2}(t)+\left[M\left(z_{2}\right)-M(z)\right] a_{1}(t) \\
& \left.+\int_{z,}^{z_{2}} K(z, a) I(a, t) d z\right\}
\end{aligned}
$$

where

and

$$
\left.-V_{y}(a, t)\right] \text { ) }
$$

$$
K(z, \mu)= \begin{cases}{\left[M(s)-M\left(z_{1}\right)\right]\left[M(z)-M\left(z_{2}\right)\right]} & z \geq s . \\ {\left[M(z)-M\left(z_{1}\right)\right]\left[M(s)-M\left(z_{2}\right)\right]} & z \leq s .\end{cases}
$$

The Fredholm series solution of (A5) (see e.g. Margenau and Murphy, 1956) converges reasonably well for $\quad W=0.7 \times 10^{-4} \mathrm{sec}^{-1}$.

A perturbation solution to (11) may also be found by taking $V \operatorname{rg}(z, t)$ $=\sum_{n=0}^{\infty} B^{n} \sim_{n}(z, t)$ with

a positive constant and $v_{0}(z, t)$ being the solution $\bar{V}_{y}(z, t)$ to (18). The equation for $n=1$ is

$$
\rho\left(\partial v_{i} / \partial t\right)+\left(B_{0}^{2} o_{p} / c^{2}\right) w_{1}=\partial / \partial z\left(\mu\left(\partial v_{0} / \partial z\right)\right)
$$

etc. This approach is useful when viscous forces are not too important. 
Margenau, H. and G. M. Murphy, The Mathematics of Physics and Chemistry,

D. Van Nostrand Company, Princeton, New Jersey, 1956.

Tölke, F., Besselsche und Hankelsche Zylinderfunktionen Nullter Bis Dritter Ordnung

Vom Argument $\Omega \sqrt{i}$, Verlag Von Konrad Wittwer, Stuttgart, 1936.

Young, A. and A. Kirk, Royal Society Mathematical Tables Volume 10, Bessel

Functions Part IV, Kelvin Functions, The University Press, Cambridge, 1964. 


\section{REFERENCES}

Axford, W. I., Viscous Interaction Between the Solar Wind and The Earth's Magnetosphere, Planet. Space Sci.,12, 45-54, 1964.

Axford, W. I. and C. O. Hines, A Unifying Theory of High Latitude Geophysical

Phenomena and Geomagnetic Storms, Can. J. Phys.,39, 1433-1464, 1961.

Boström, R., A Model of Auroral Electrojets, J. Geophys. Res.,69, 4983-4999, 1964.

Chapman, S., The Electrical Conductivity of the lonosphere: A Review, Nuovo

Cimento, Suppl., 4, 1385-1412, 1956.

Chapman, S. and T. G. Cowling, The Mathematical Theory of Non-Uniform Gases,

Cambridge University Press, Cambridge, 1960.

Fejer, J. A., Theory of the Geomagnetic Daily Disturbance Variations, J. Geophys. Res.,69, 123-137, 1964.

Gold, T., Motions in the Magnetosphere of the Earth, J. Geophys. Res.,64, 1219-1224, 1959.

Hanson, W. B., Structure of the lonosphere, in Satellite Environment Handbook,

Edited by F. S. Johnson, pp. 25-46, Stanford University Press, Stanford, 1961.

Hines, C. O., Hydromagnetic. Motions in the Magnetosphere, Space Science Reviews,3, 342-379, 1964.

Johnson, F. S., Chapters 1 and 2 of Satellite Environment Handbook, Edited by F. S. Johnson, pp. 9-46, Stanford University Press, Stanford, 1961.

Levy, R. H., H. E. Petschek and G. L. Siscoe, Aerodynamic Aspects of the Magnetospheric Flow, Avco Everett Research Laboratory Report 170, December, 1963. 
TABLE 1

The ratio

$$
S\left(z_{2}, t\right) / V_{p o}
$$$$
=\tilde{C} \sin (\omega t+\tilde{\theta})
$$

(dynes-sec $/ \mathrm{cm}^{3}$ ) for four cases. $\widetilde{C}$ and $\tilde{\theta}$ denote the approximate values of $C$ and $\theta$ found via the variational technique.

1)

$$
\begin{gathered}
\sigma_{p o}=1 \times 10^{-17} \text { EMU, } \quad H=10 \mathrm{~km} \\
S(300 \mathrm{~km}, t) / V_{p o}=3.54 \times 10^{-11} \sin (\omega t+0.21)
\end{gathered}
$$

2)

$$
\sigma_{p 0}=1 \times 10^{-17} \text { EMU, } \quad H=25 \mathrm{~km}
$$

$$
\widetilde{C}>4.24 \times 10^{-11} \quad\left(Z_{2}=475 \mathrm{~km}\right)
$$

3)

$$
\sigma_{p o}=3 \times 10^{-15} \mathrm{EMU}, \quad H=10 \mathrm{~km}
$$

$S(300 \mathrm{~km}, t) / V_{p o}=4.81 \times 10^{-9} \sin (w t+0.19)$

4)

$$
\sigma_{p 0}=3 \times 10^{-15} \text { EMU, } \quad H=25 \mathrm{~km}
$$

$S(300 \mathrm{~km}, t) / V_{p o}=1.17 \times 10^{-8} \sin (\omega t+0.25)$ 

1)
$\sigma_{p_{0}}=10^{-17}$ EMU,
$D_{T}=110 \mathrm{~km} ;$
$\underline{Z}_{2}=450 \mathrm{~km}$
2)
$\sigma_{p_{0}}=10^{-16} \mathrm{EMU}, \quad D_{T}=35 \mathrm{~km}, \quad Z_{2}=400 \mathrm{~km}$
3)

$$
\sigma_{p o}=3 \times 10^{-15} \mathrm{EMU} \text {, }
$$
$D_{T}=6.33 \mathrm{~km}$,
$Z_{2}=300 \mathrm{~km}$

Fig. $7 \quad-10^{8} \times\left(b_{y}(z) / V_{p o} \quad\right.$ Gauss-sec/cm) versus $z$ (Eqn. 16b).

Curves 1, 2, and 3 are drawn for the parameter values described under Fig. 6 .

Fig. 8 The Pederson conductivity $\sigma_{p}(z)$ (EMU) for various conditions.

For $\quad z \geq 100 \mathrm{~km}$ these conductivity curves are taken from Hanson (1961).

For $\quad z<100 \mathrm{~km}$ the conductivities were calculated from the formulae and data of Hanson (1961).

Fig. 9 Pederson conductivity $\sigma_{p}$ (EMU) versus $Z$; approximate representations of the four curves of Fig. 8.

Fig. 10 The mass density $\rho(z)$ versus height for sunspot maximum and minimum (from Johnson, 1961) and for two values of $H$.

Fig. $11 g_{A}, \bar{g}_{A}, \widetilde{g_{A}}$, and $g$ versusheight. $g_{A}$ is the exact solution including viscosity and $g=V_{y}(z) / V_{p o}$ with $V_{y}(z)$ given by $(16 a)$.

Fig. $12 g_{B}, \overline{g_{B}}, \tilde{g}_{B}$ versus height. $g_{B}$ is the exact solution including viscosity.

Fig. 13 $\bar{g}_{A}$ and $\bar{g}_{B}$ (solid lines) versus height. These curves were determined for $W=0.6 \times 10^{-3} \sec ^{-1}$ from the $\rho$ for sunspot maximum (Fig. 10) and the $\sigma_{p}$ for day, sunspot maximum (Fig. 8). The curves $g_{A}, g_{B}$, including viscosity, lie with in the dashed lines. 


\section{FIGURE CAPTIONS}

Fig. 1 Gross features, shown in the equatorial plane of the magnetosphere, of the convection pattern suggested by Axford and Hines. The feet of the convecting flux tubes extend down to ionospheric levels. Geographic and geomagnetic axes are assumed to coincide. The region $R$ inside the dashed circle around the earth is the region of a possible inner convection system. The earth is drawn disproportionately large.

Fig. 2 The streamlines of convective flow obtained by mapping down into the north polar ionosphere the flow pattern indicated in Fig. 1, (adapted from Axford and Hines, 1961). This pattern is intended to represent moderately disturbed conditions.

Fig. 3 The velocities of Fig. 2 as seen by observers rotating with the earth. The velocity in the high velocity regions $E, F$ and $G, H$ is about $1 \mathrm{~km} / \mathrm{sec}$.

Fig. 4 A cross section of the model in the $x, z$ plane. The solid lines are the electric field lines and the dashed lines are the flow lines of the current $\overrightarrow{y_{P}} \cdot \vec{E}$ and $\overrightarrow{y_{P}}$ vary sinusoidally in time.

Fig. 5 A finite section of the (infinitely long) model, viewed in the $y, z$ plane. The velocities $\overrightarrow{V P}$ and $\vec{V}$ lie in the $y$ direction as does the current $\vec{\gamma}_{N}$. The perturbed magnetic field, with its departure from the vertical considerably exaggerated, is also shown.

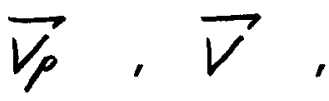
$\vec{f}_{A}$, and the perturbation field all vary sinusoidally in time.

Fig. 6

$$
\begin{array}{ll} 
& V_{y}(z) / V_{p o} \text { versus } z \quad(\text { Eqn. 16a). For all } \\
\text { three curves } & B_{0}=0.5 \text { gauss, } \quad \mu_{0}=3 \times 10^{-4} \mathrm{~g} / \mathrm{cm}-\mathrm{sec} \text {, and }
\end{array}
$$$$
Z_{1}=80 \mathrm{~km} \text {. For curve }
$$ 


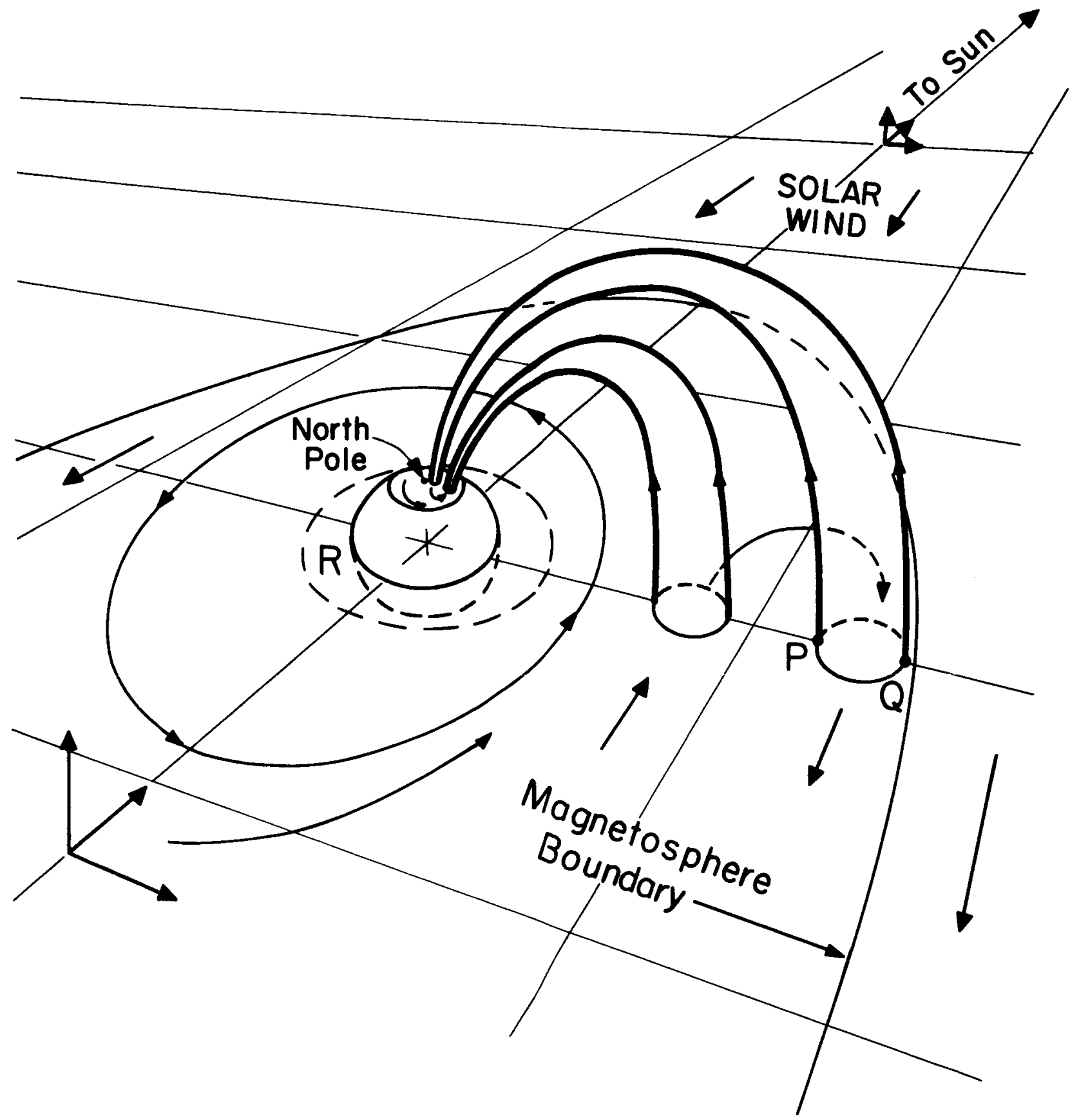

Fig. I 


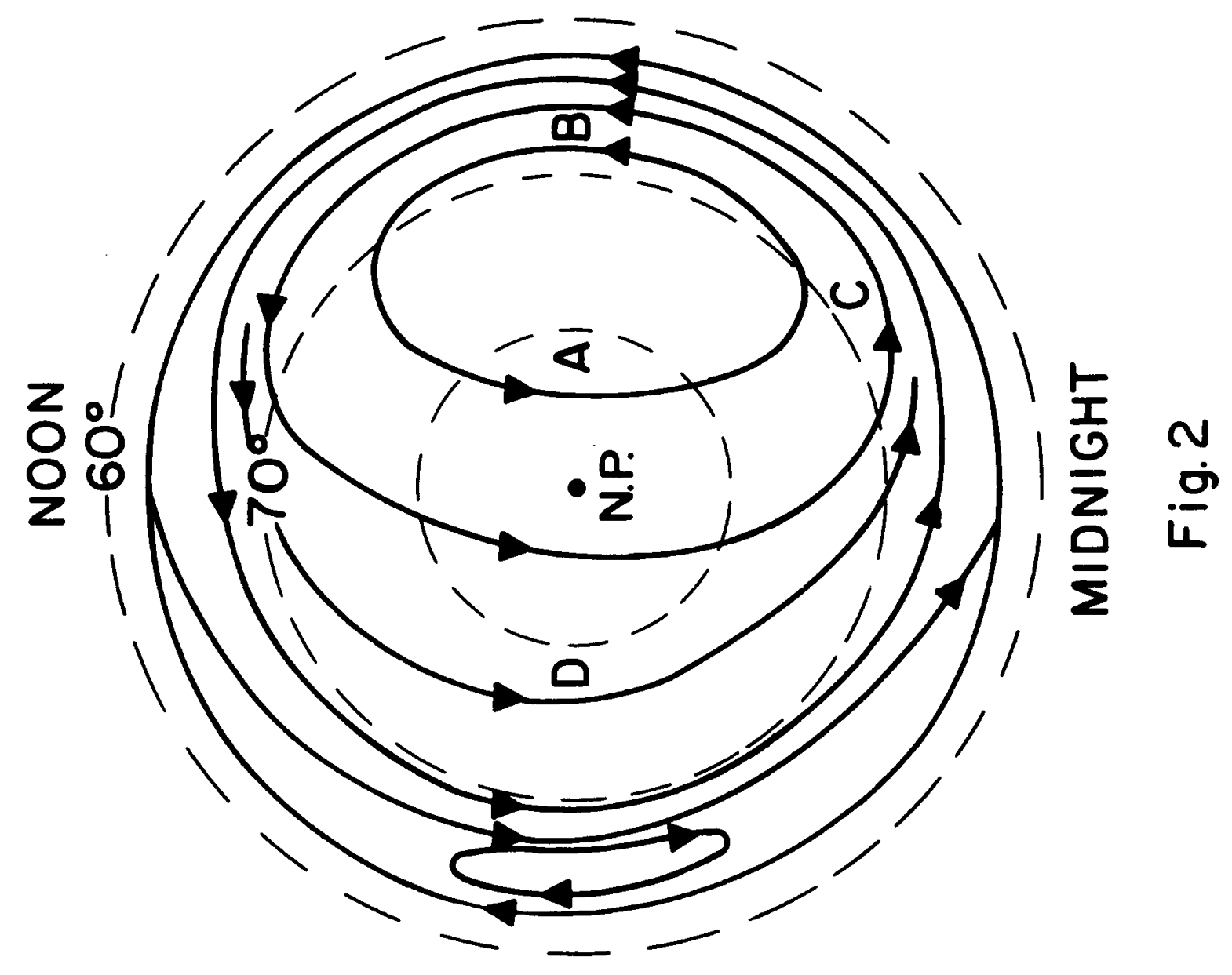




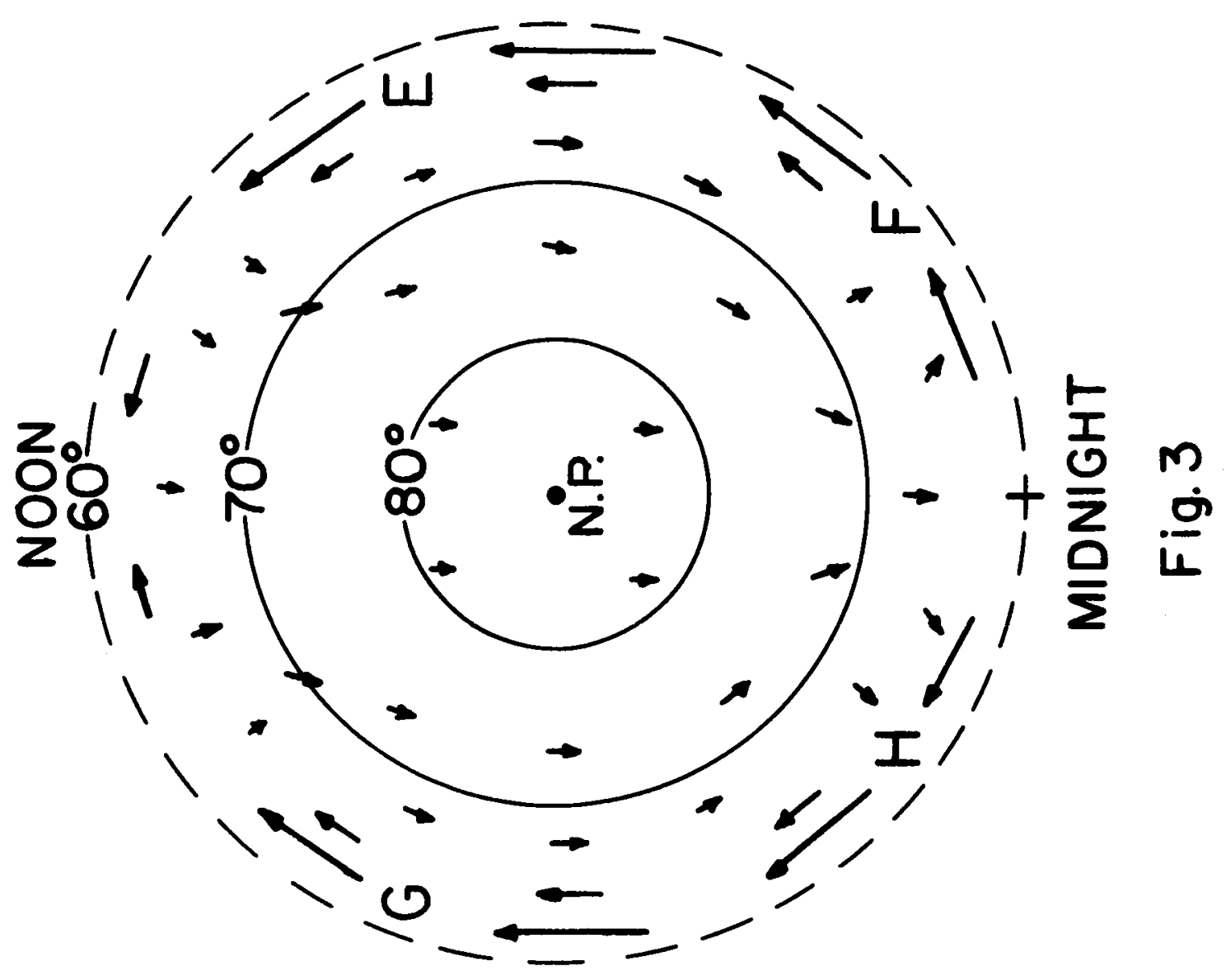




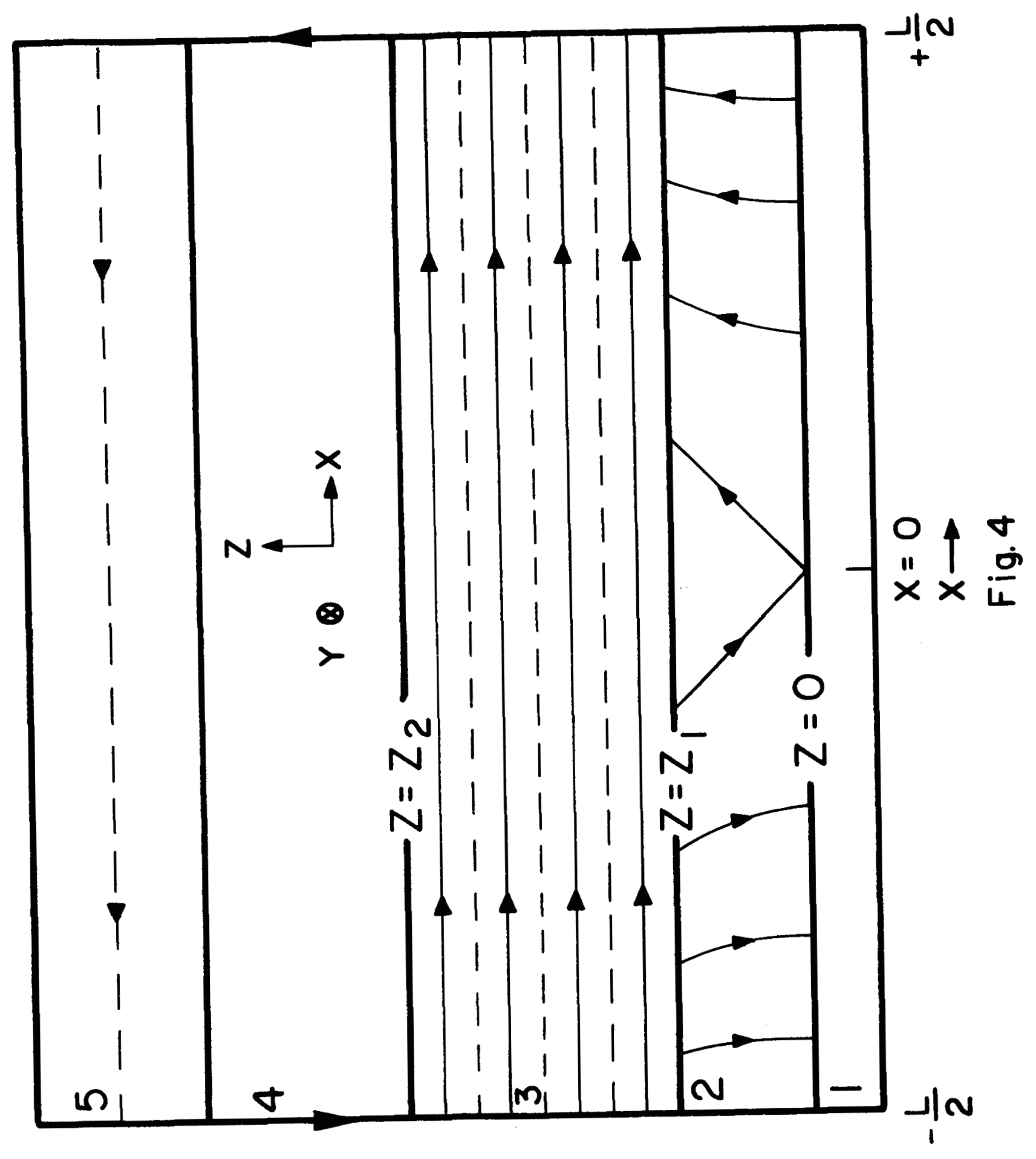




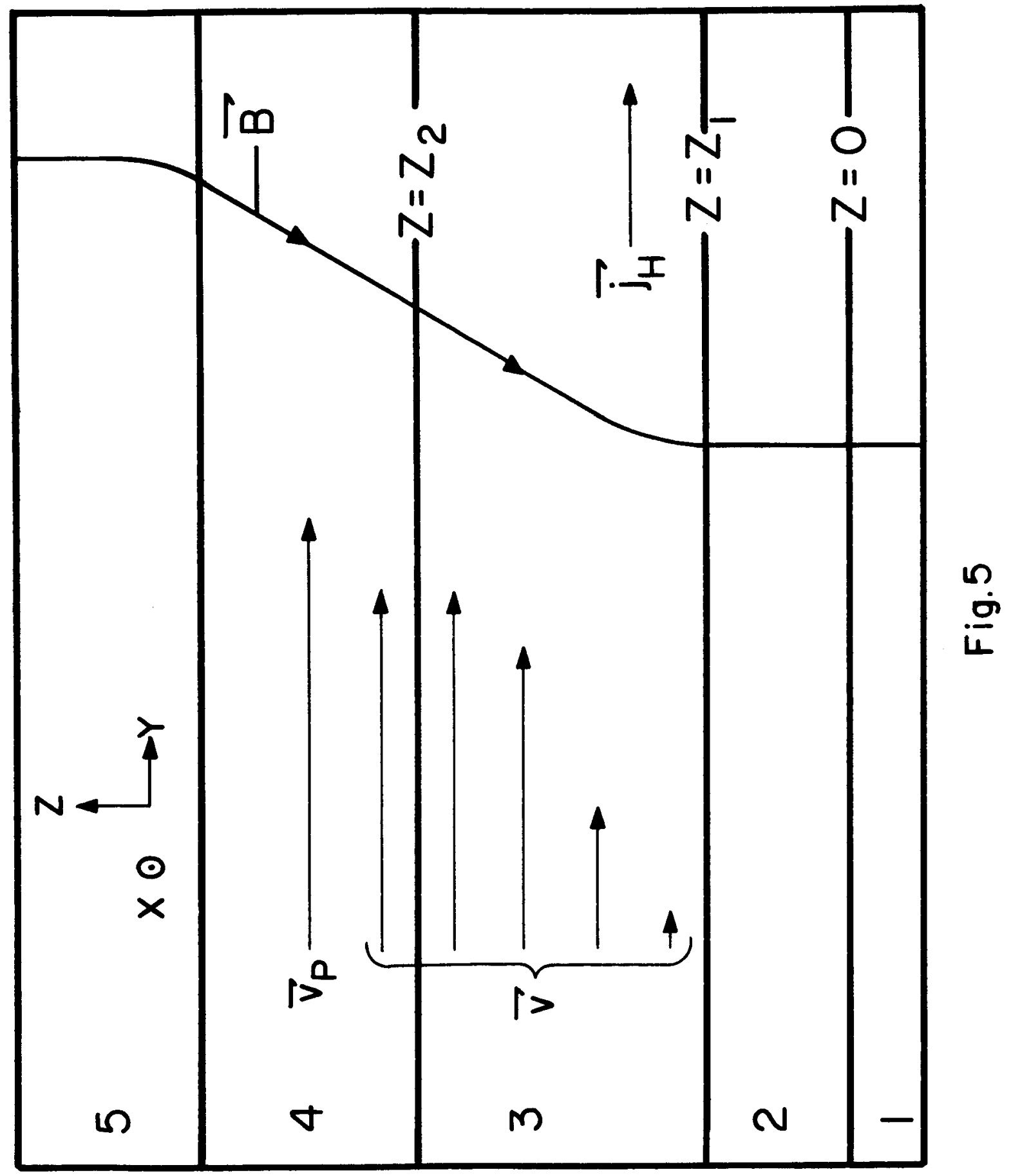




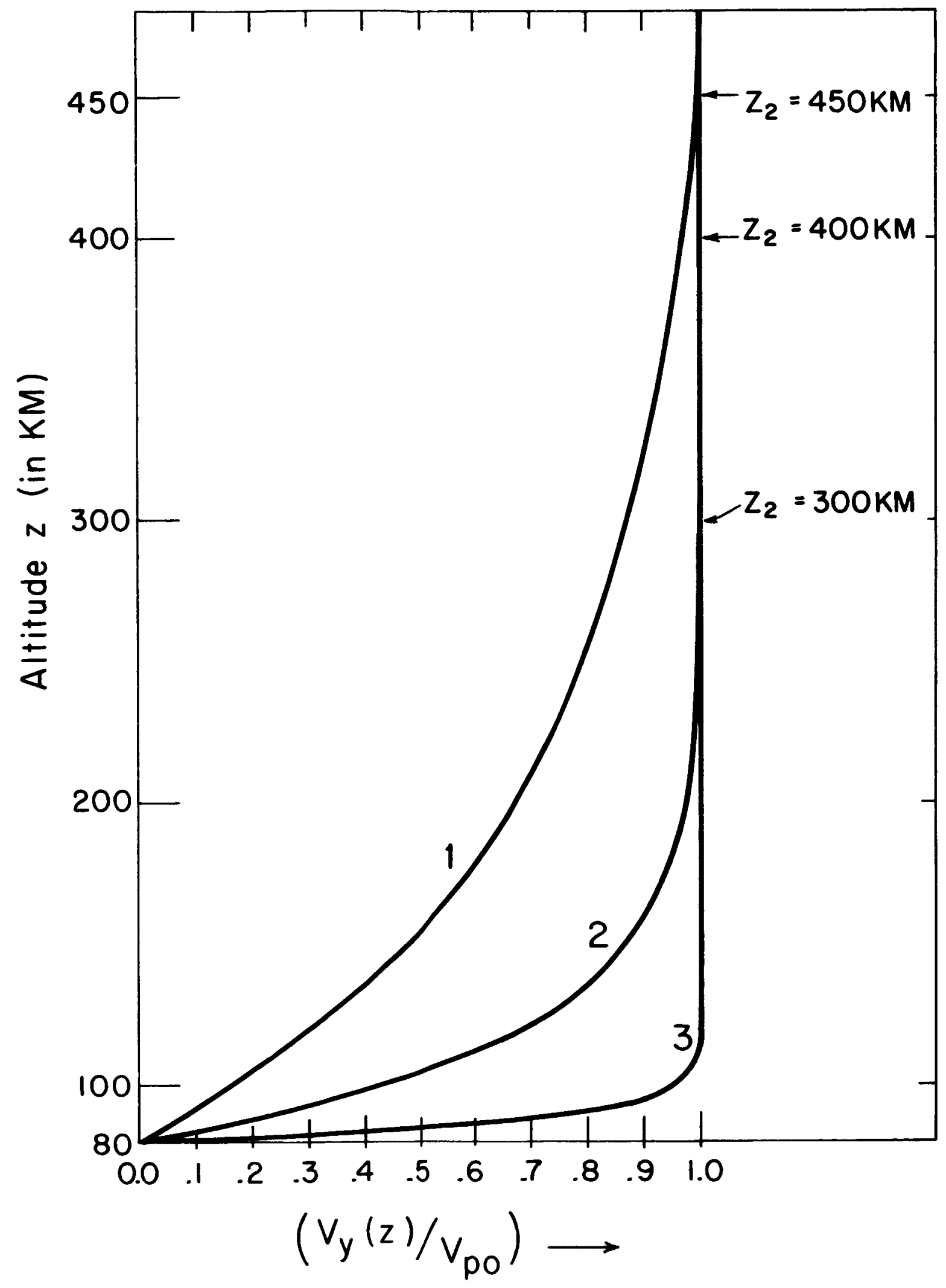

Fig. 6 


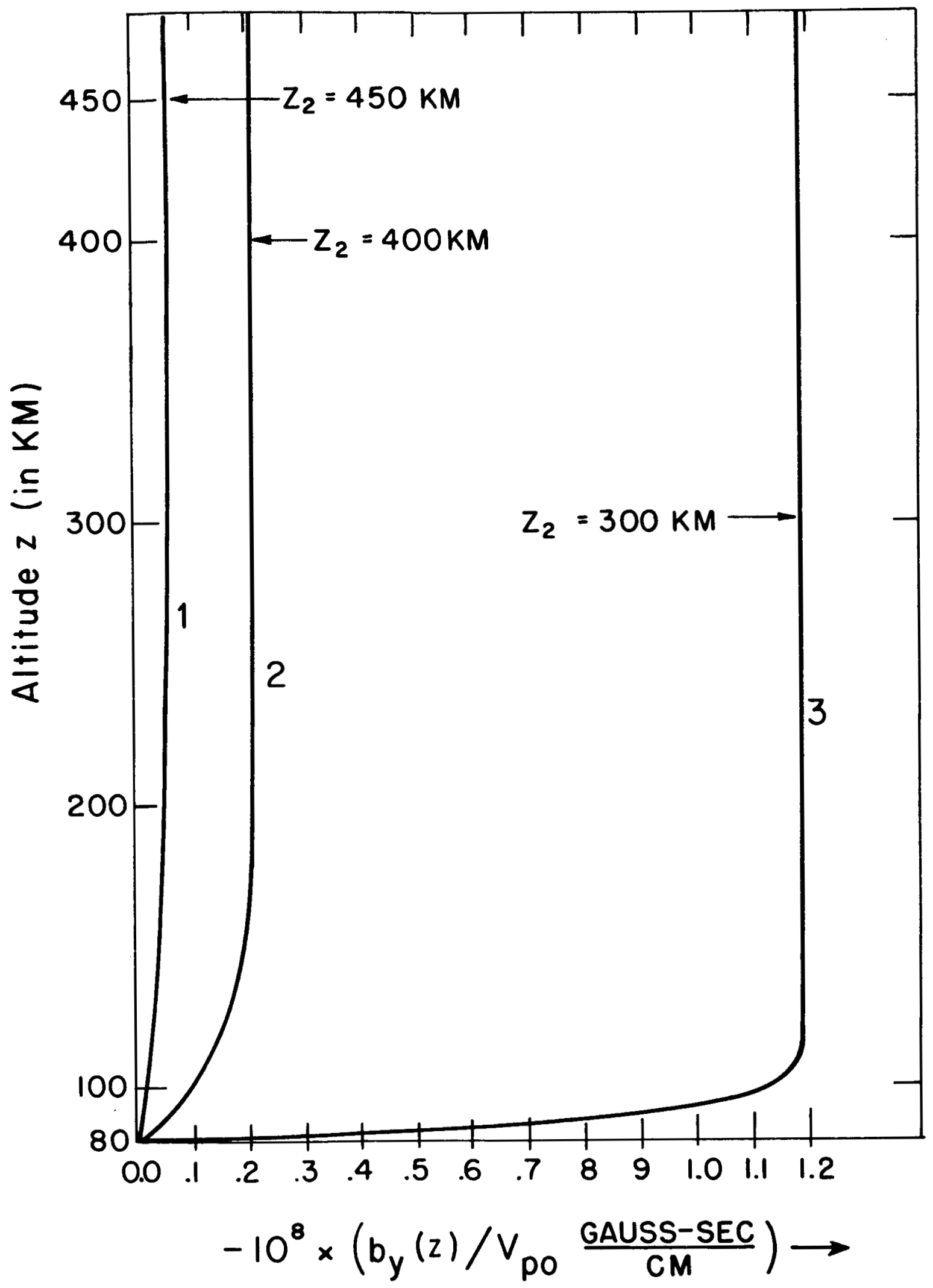

Fig. 7 


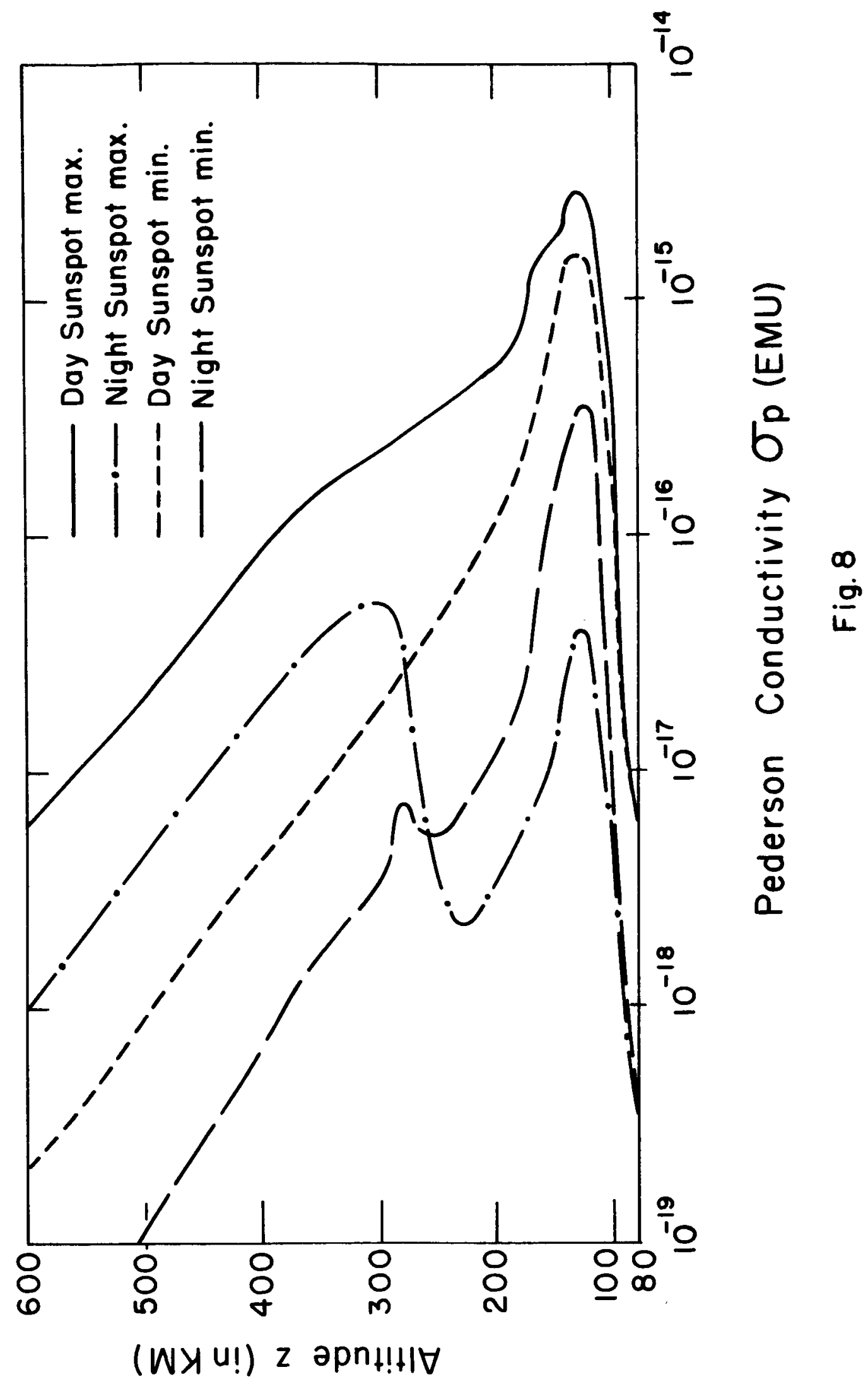




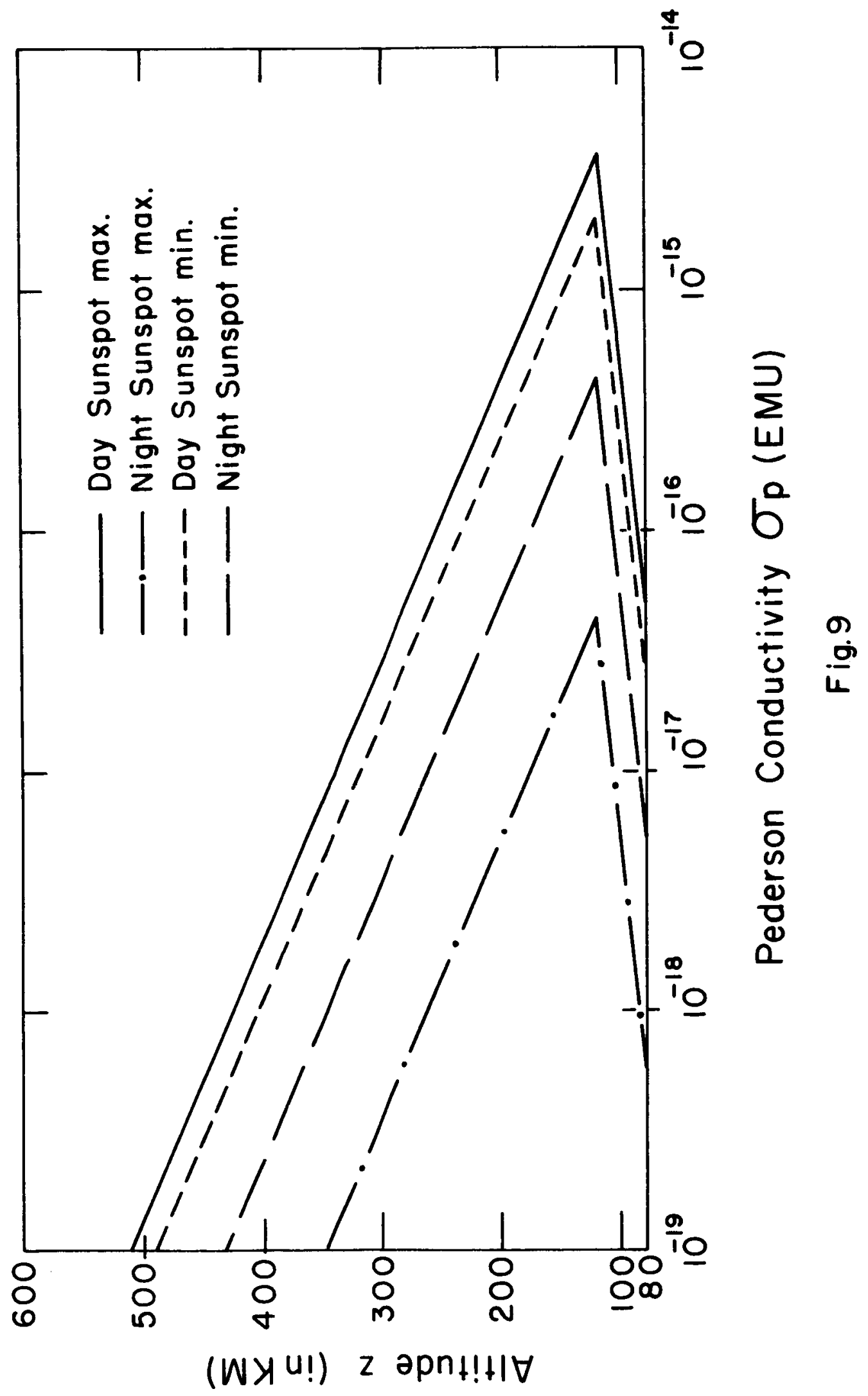




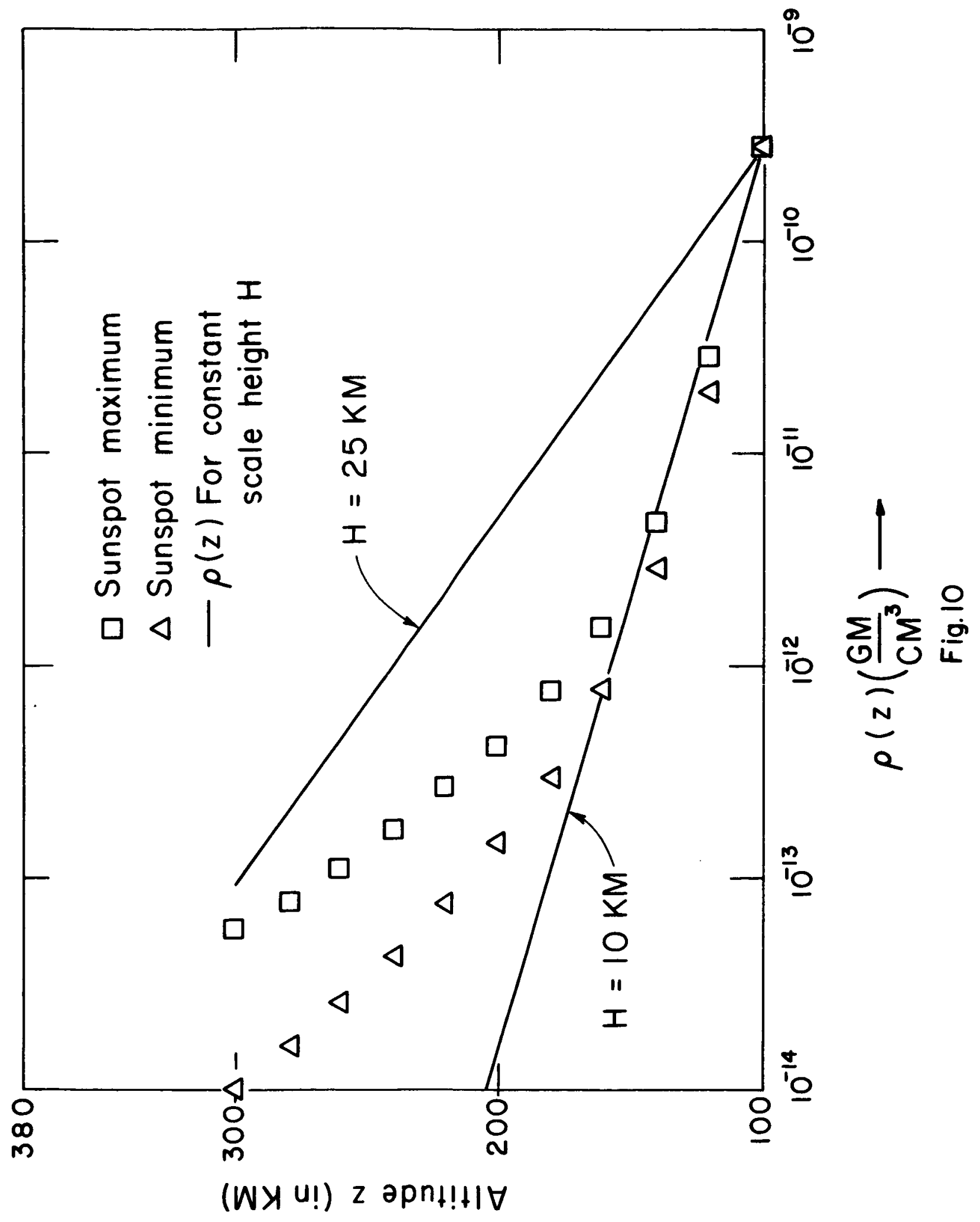




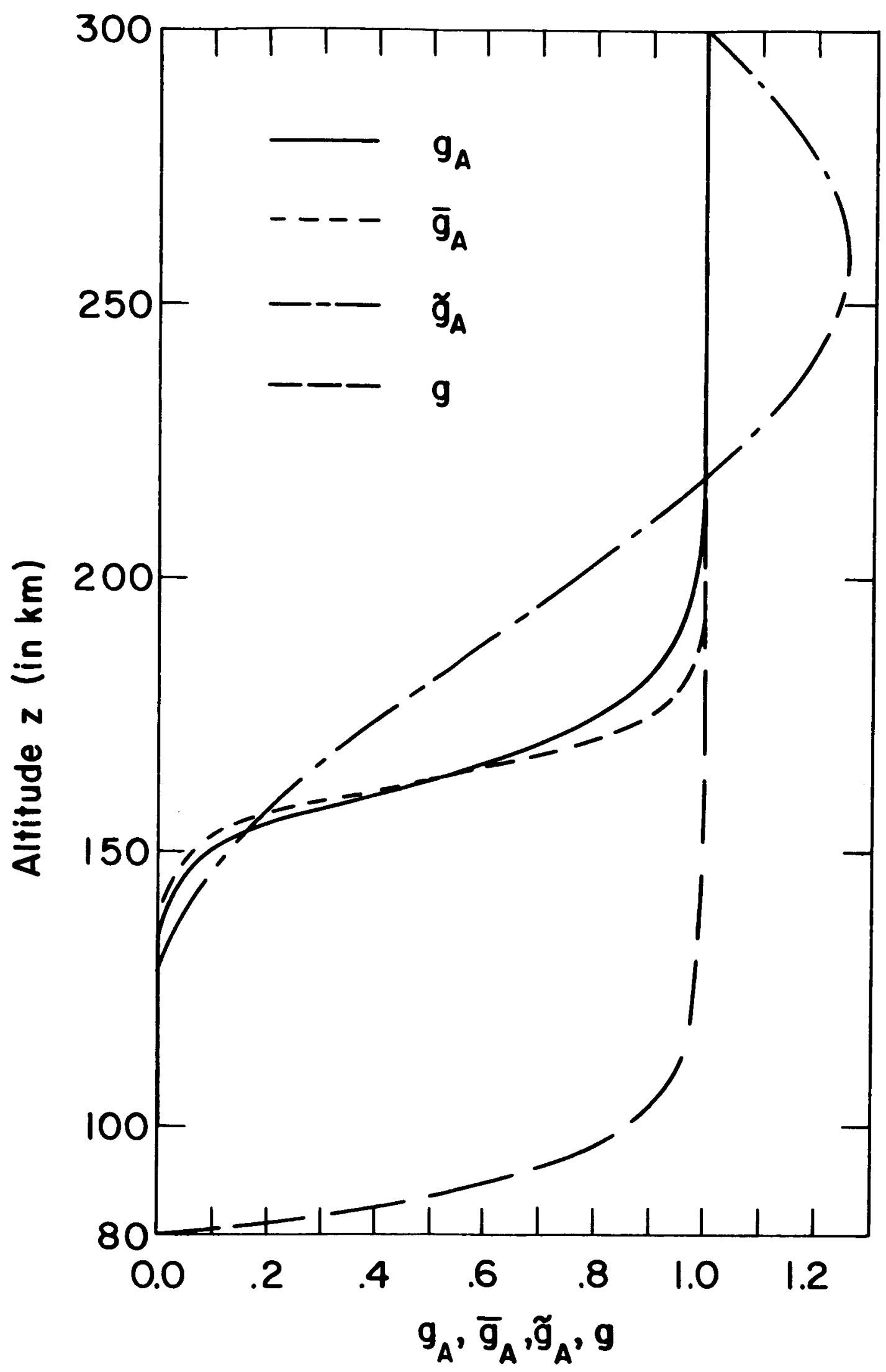

Fig.II 


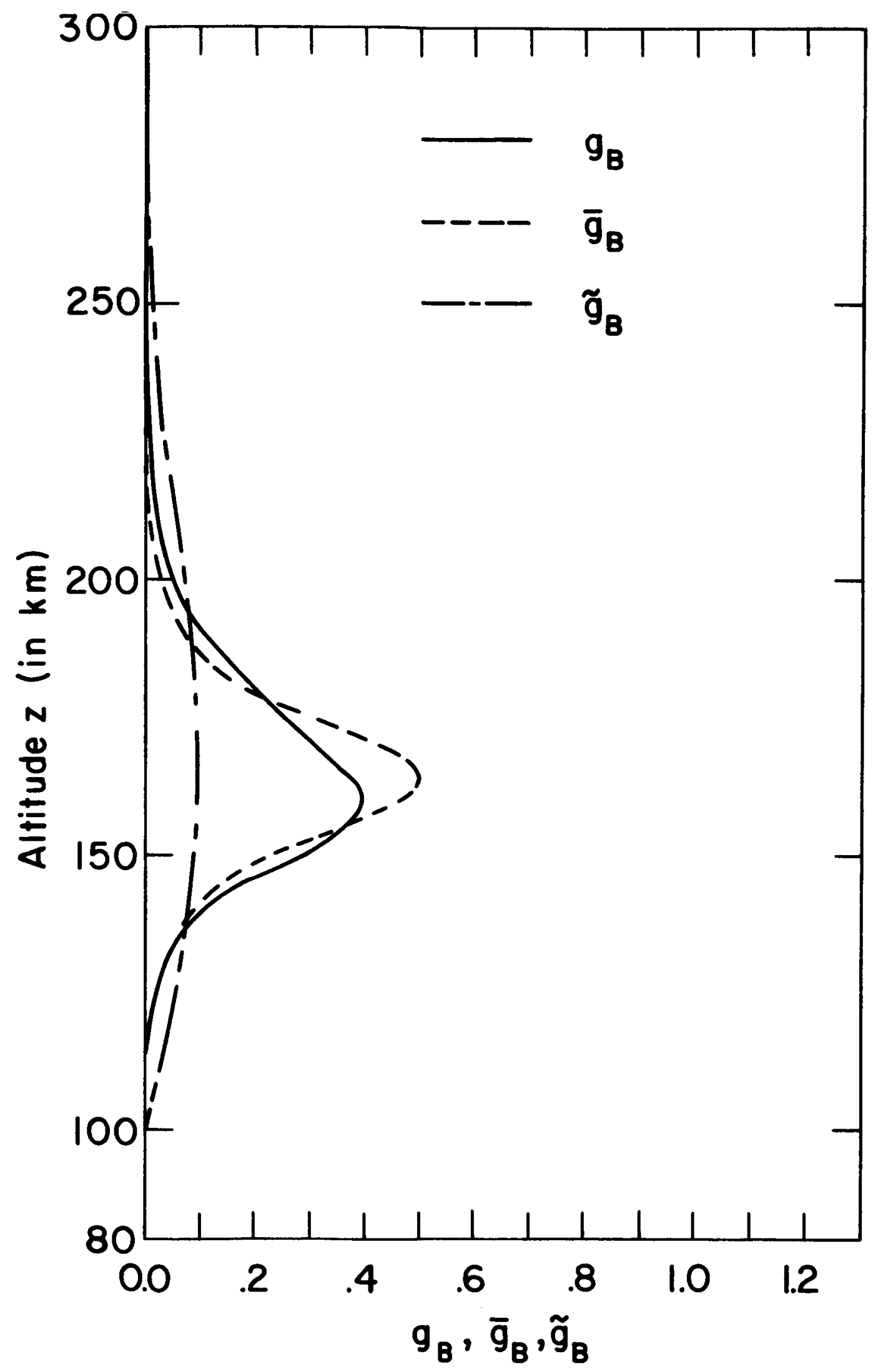

Fig. 12 


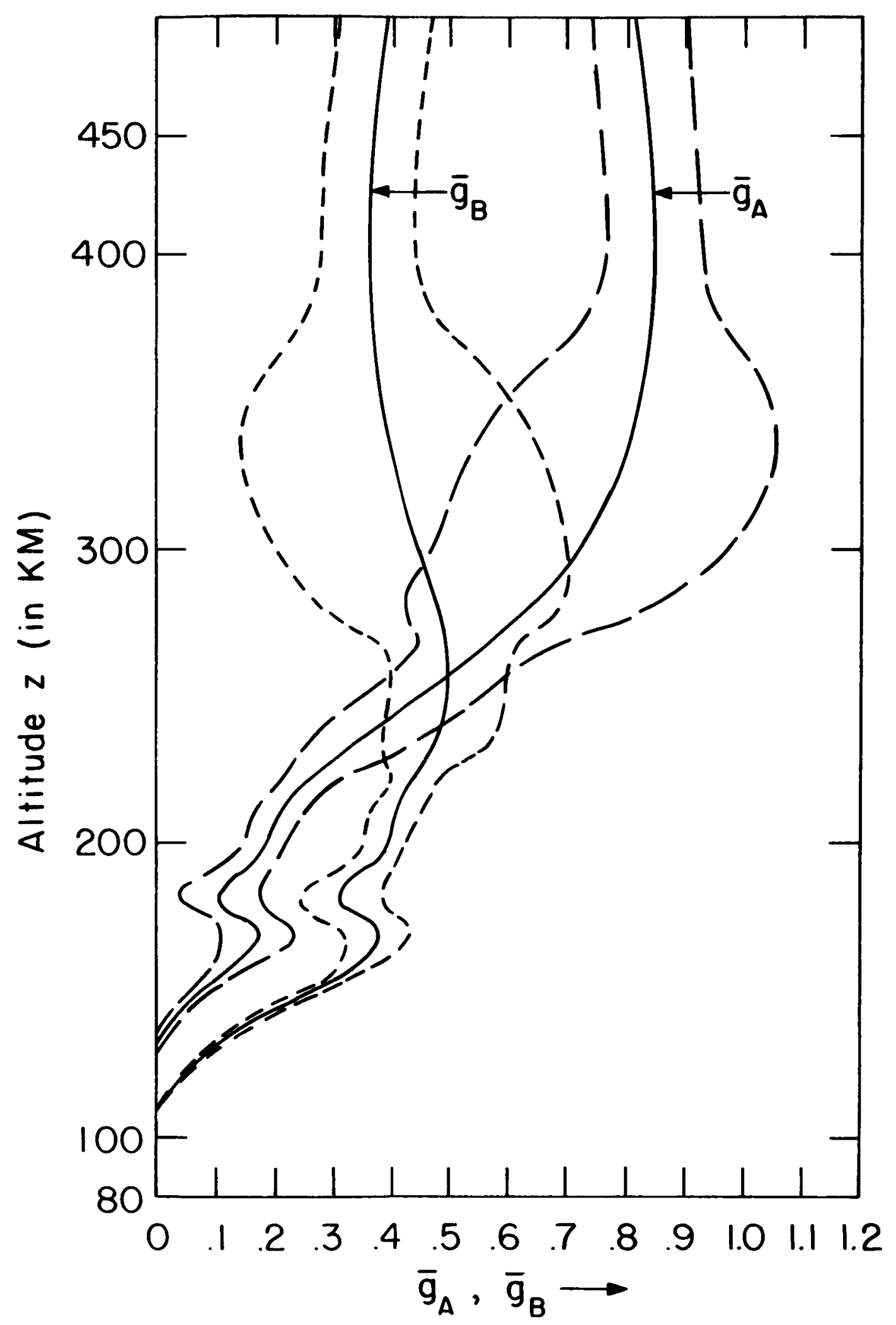

Fig. 13 